\title{
COISOTROPIC REPRESENTATIONS OF REDUCTIVE GROUPS
}

\author{
I. V. LOSEV
}

\begin{abstract}
A symplectic action $G: X$ of an algebraic group $S$ on a symplectic algebraic variety $X$ is called coisotropic if a generic orbit of this action is a coisotropic submanifold of $X$. In this article a classification of coisotropic symplectic linear actions $G: V$ is given in the case where $G$ is a reductive group.
\end{abstract}

\section{INTRODUCTION}

Let $G$ be a reductive complex algebraic group, $X$ a symplectic smooth affine algebraic manifold, and $G: X$ a symplectic action. The action $G: X$ is called coisotropic if one of the following equivalent conditions holds (the proof of equivalence is similar to the proof in [3, Chapter 2, Section 3.2, Proposition 5]):

(CO1) A generic orbit of the action $G: X$ is coisotropic.

(CO2) The restriction of the Poisson bracket to the algebra $\mathbb{C}(X)^{G}$ of rational invariants is commutative.

Condition (CO1) can also be rewritten as follows:

(CO3) $m_{G}(X)+\operatorname{def}_{G}(X)=\operatorname{dim} X$.

It is clear that the action $G: X$ is coisotropic if and only if the action $G^{\circ}: X$ of the connected component of unity is coisotropic. Therefore, in this paper we consider only connected groups $G$.

The goal of the paper is to classify coisotropic linear actions (coisotropic representations) $G: V$, where $V$ is a symplectic vector space and $G$ is a reductive group. In the case where $G$ is a subgroup of the group $\operatorname{Sp}(V)$, we will say that a linear group $G$ is coisotropic if the representation $G: V$ is coisotropic.

The answer to the question of whether a representation $G: V$ is coisotropic is known in the case where the $G$-module $V$ is isomorphic to $U \oplus U^{*}$ for some $G$-module $U$. The symplectic form $\omega$ on the space $U \oplus U^{*}$ is defined as follows: for $u^{*} \in U^{*}, u \in U$, we have $\omega\left(u^{*}, u\right)=u^{*}(u)$, and the restrictions of $\omega$ to $U$ and to $U^{*}$ vanish. Namely (see, e.g., Theorem 2 in Chapter 2 of [3]), the representation $G: U \oplus U^{*}$ is coisotropic if and only if the action $G: U$ is spherical, i.e., the Borel subgroup $B \subset G$ has a dense orbit in $U$. Spherical linear actions are classified: the case where the representation $G: U$ is irreducible is considered in [9], and the general case in, say, 10].

One can show (see, e.g., Proposition 6) that symplectic direct summands of a coisotropic representation are coisotropic as well. Therefore, the first step in the classification of all coisotropic representations is the classification of irreducible coisotropic representations. The results of this classification are presented in Table 1.

An explicit description of reducible coisotropic representations would take too much space. However, one can describe "basic" coisotropic representations, from which all the

2000 Mathematics Subject Classification. Primary $20 \mathrm{C} 15$. 
TABLE 1. Irreducible coisotropic representations.

\begin{tabular}{|c|c||c|c|}
\hline & $G$ & & $G$ \\
\hline 1 & $\operatorname{Sp}(2 l)$ & 2 & $\bigwedge^{3} \mathrm{SL}(6)$ \\
\hline 3 & $\operatorname{Spin}(11)$ & 4 & $\operatorname{Spin}(13)$ \\
\hline 5 & $C_{3}\left(\pi_{3}\right)$ & 6 & $\operatorname{Spin}_{ \pm}(12)$ \\
\hline 7 & $E_{7}\left(\pi_{1}\right)$ & 8 & $\mathrm{Sp}(2 m) \otimes S O(n)$ \\
\hline 9 & $\mathrm{Sp}(2 m) \otimes \operatorname{Spin}(7)$ & 10 & $\mathrm{SL}(2) \otimes \operatorname{Spin}(9)$ \\
\hline 11 & $\mathrm{SL}(2) \otimes G_{2}\left(\pi_{1}\right)$ & 12 & $\mathrm{Sp}(4) \otimes G_{2}\left(\pi_{1}\right)$ \\
\hline 13 & $S^{3} \mathrm{SL}(2)$ & & \\
\hline
\end{tabular}

other coisotropic representations can be easily constructed. To formulate the corresponding result we need some terminology.

A symplectic linear group is called decomposable if it can be represented as a direct sum of two symplectic linear groups. Otherwise, the group is called indecomposable. The classification of all coisotropic representations is trivially reduced to the classification of indecomposable coisotropic representations.

A reductive linear group $G \subset \operatorname{Sp}(V)$ (or the corresponding representation) is called saturated if $Z_{\mathrm{Sp}(V)}(G)=Z(G)$ (i.e., if the group $G$ has the maximal possible center for the given semisimple part $\left.G^{\prime}\right)$. For a given reductive linear group $G \subset \operatorname{Sp}(V)$ there exists a saturated symplectic linear group $\widetilde{G} \subset \operatorname{Sp}(V)$ such that $(\widetilde{G}, \widetilde{G}) \subset G \subset \widetilde{G}$. The group $\widetilde{G}$ is called the saturation of the group $G$. Let us note that if the group $G$ is indecomposable, then its saturation $\widetilde{G}$ is indecomposable as well. On the other hand, the converse statement does not always hold. In principle, the classification of indecomposable coisotropic representations can be reduced to the classification of coisotropic representations with indecomposable saturation (see Proposition 12). Similarly one defines nonsaturated and saturated symplectic representations.

The next statement allows us to restrict our attention to an even smaller class of coisotropic representations. Let $\widehat{G}=\mathrm{SL}(2) \times \mathrm{SL}(2) \times G_{0}$ be a reductive algebraic group and $\rho$ a coisotropic locally exact symplectic representation of the group $\widehat{G}$. Let us consider the subgroup $G=\operatorname{SL}(2) \times G_{0} \subset \widehat{G}$, where the factor $\operatorname{SL}(2) \subset G$ is embedded in $\operatorname{SL}(2) \times$ $\mathrm{SL}(2) \subset \widehat{G}$ diagonally. Representations of the form $\left.\rho\right|_{G}$ are called $A_{1}$-glued, and all other representations, $A_{1}$-nonglued. Similar terminology is used for symplectic linear actions. Proposition 13 in Subsection 2.4 reduces the classification of all actions to the classification of $A_{1}$-nonglued actions.

In Table 2 we present the commutator subgroups $G^{\prime}$ of all $A_{1}$-nonglued saturated indecomposable coisotropic linear groups $G$ except the groups $G$ of the form $H^{\text {sympl }}$. Notation is explained in Subsection 2.4 after Table 10.

Now we briefly describe the content of this paper. The text consists of sections, which have subsections, and, sometimes, subsubsections.

In Section 1 we present the main tools used for classification. In Subsection 1.1 we recall the main properties of the moment map. Knowing a generic stabilizer of the action $G: V$ and the stabilizer of a generic element in the image of the moment map allows one to determine whether or not the representation $G: V$ is coisotropic. A particularly simple case occurs when the image of the moment map contains a (Zariski) open subset of the space $\mathfrak{g}^{*} \cong \mathfrak{g}$ consisting of semisimple elements of the algebra $\mathfrak{g}$. Following Vinberg [3] we say that such representations are symplectically stable. In this 
TABLE 2. $A_{1}$-nonglued saturated indecomposable coisotropic linear groups $G$.

\begin{tabular}{|c|c|}
\hline & $G^{\prime}$ \\
\hline 1 & $A_{5}\left(\pi_{3}, \pi_{1}, \pi_{5}\right)$ \\
\hline 2 & $C_{l}\left(\pi_{1}, \pi_{1}, \pi_{1}\right)$ \\
\hline 3 & $C_{3}\left(\pi_{3}, \pi_{1}, \pi_{1}\right)$ \\
\hline 4 & $B_{2}\left(\pi_{2}, \pi_{1}, \pi_{1}\right)$ \\
\hline 5 & $D_{6}\left(\pi_{5}, \pi_{1}, \pi_{1}\right)$ \\
\hline 6 & $\operatorname{Spin}(12)$ \\
\hline 7 & $\wedge^{3} \mathrm{Sp}(6)$ \\
\hline 8 & $(\mathrm{SL}(2) \oplus \mathrm{SL}(2)) \otimes \mathrm{SO}(n), n>4$ \\
\hline 9 & $\mathrm{Sp}(2 m)+_{\mathrm{Sp}(2 m)} \mathrm{Sp}(2 m) \otimes \mathrm{SO}(n), m>1, n>2$ \\
\hline 10 & $\operatorname{Sp}(4)+\operatorname{Sp}(4) \operatorname{Sp}(4) \otimes \operatorname{Spin}(7)$ \\
\hline 11 & $(\mathrm{SL}(2) \oplus \mathrm{SL}(2)) \otimes \operatorname{Spin}(7)$ \\
\hline 12 & $\mathrm{SL}(2) \otimes \mathrm{SO}(11)+{ }_{\mathrm{SO}(11)} \mathrm{Spin}(11)$ \\
\hline 13 & $\mathbf{S p}(\mathbf{2 m}) \otimes \mathrm{SO}(12)+\mathrm{SO}(12) \operatorname{Spin}_{ \pm}(12), m=1,2$ \\
\hline 14 & $\mathrm{Sp}(4)+{ }_{\mathrm{Sp}(4)} \mathrm{SO}(5) \otimes \mathbf{S p}(\mathbf{2 m})$ \\
\hline 15 & $\mathrm{SL}(2) \otimes \mathrm{SO}(7)+{ }_{\mathrm{SO}(7)} \operatorname{Spin}(7) \otimes \mathbf{S L}(\mathbf{2})$ \\
\hline 16 & $\mathbf{S L}(2) \otimes \mathbf{S O}(8)+{ }_{\mathrm{SO}(8)} \operatorname{Spin}_{+}(8) \otimes \mathbf{S L}(\mathbf{2})$ \\
\hline 17 & $\mathrm{SL}(2) \otimes \mathrm{SO}(8)+\mathrm{SO}(8) \operatorname{Spin}_{+}(8) \otimes \mathrm{Sp}(4)$ \\
\hline 18 & $\wedge^{3} \mathrm{SL}(6)+\mathrm{SL}(6)(\mathrm{SL}(6) \otimes \mathbf{S L}(\mathbf{2}))^{\mathrm{sympl}}$ \\
\hline 19 & $\mathrm{SL}(2) \otimes \mathrm{SO}(5)+{ }_{\mathrm{SO}(5)} \mathrm{Sp}(4)^{\mathrm{sympl}}$ \\
\hline 20 & $\mathbf{S L}(\mathbf{2}) \otimes \mathrm{SO}(6)+{ }_{\mathrm{SL}(4)} \mathrm{SL}(4)^{\mathrm{sympl}}$ \\
\hline 21 & $\mathrm{SL}(2) \otimes \mathrm{SO}(7)+{ }_{\mathrm{SO}(7)} \operatorname{Spin}(7)^{\mathrm{sympl}}$ \\
\hline 22 & $\mathbf{S L}(2) \otimes \mathbf{S O}(8)+{ }_{\mathrm{SO}(8)} \operatorname{Spin}_{ \pm}(8)^{\mathrm{sympl}}$ \\
\hline 23 & $\mathbf{S L}(\mathbf{2}) \otimes \mathbf{S O}(10)+_{\mathrm{SO}(10)} \operatorname{Spin}_{ \pm}(10)^{\mathrm{sympl}}$ \\
\hline
\end{tabular}

case the property of $G: V$ to be coisotropic is determined by a generic stabilizer of the representation $G: V$; in many cases this stabilizer can be found using results of 1, 6, 7. In Subsection 1.2 we give the list of all representations that are not symplectically stable (Theorem 11). In Subsection 1.3 we prove that for all symplectic actions of reductive groups the properties of symplectic stability, stability (i.e., the closedness of a generic orbit), and the reductivity of a generic stabilizer are equivalent. In Subsection 1.4 we discuss the structure of reducible coisotropic representations.

In Section 2 we present the results of the classification of coisotropic representations. Subsections 2.1 and 2.2 are devoted to irreducible coisotropic representations for simple and for nonsimple groups respectively. In Subsection 2.3 we present the results of the classification of spherical representations. Finally, in Subsection 2.4 we classify 
coisotropic representations $G: V$ such that the $G$-module $V$ is not of the form $U \oplus U^{*}$ for a $G$-module $U$.

In conclusion, the author wants to thank his advisor E. B. Vinberg for the formulation of the problem and attention to this work.

\section{SOME NOTATION}

- $G_{x}$ is the stabilizer of a point $x \in X$ for the action $G: X, \mathfrak{g}_{x}$ is the Lie algebra of the group $G_{x}$.

- $\Sigma\left(\lambda_{1}, \ldots, \lambda_{k}\right)$ is the linear group which is the image of the simply connected semisimple complex algebraic group with the Dynkin diagram $\Sigma$ under the representation with the highest weight $\lambda_{1}, \ldots, \lambda_{k}$.

- The linear groups $G \otimes H, G \oplus H, S^{k} G, \bigwedge^{k} G$ are the tensor product and the direct sum of the linear groups $G$ and $H$, the symmetric and the exterior power of the linear group $G$ respectively.

- $\mathfrak{n}_{\mathfrak{g}}(\mathfrak{h})$ is the normalizer of the subalgebra $\mathfrak{h}$ in the Lie algebra $\mathfrak{g}$.

- $N_{G}(\mathfrak{h})$ is the normalizer of the subalgebra $\mathfrak{h} \subset \mathfrak{g}$ in the group $G$.

- $Z_{G}(H)$ is the centralizer of the subgroup $H$ in the group $G$. If $H=G$ we will write $Z(G)$ instead of $Z_{G}(G)$.

- $(G, G)$ is the commutator subgroup of the group $G$.

- $\mathfrak{t}(l)$ is the $l$-dimensional commutative diagonalizable Lie algebra.

- $\operatorname{Spin}_{+}(2 m), \operatorname{Spin}_{-}(2 m), \operatorname{Spin}(2 m)$ are the images of the simply connected group of type $D_{m}$ under the semispinor and spinor representations respectively.

- $\mathfrak{s p}(2 l-1)$ is the stabilizer subalgebra of the highest vector in the tautological representation of the Lie algebra $\mathfrak{s p}(2 l)$.

- $m_{G}(X)$ is the maximal dimension of the orbit of the action $G: X$. When the manifold $X$ is clear from the context, we will write just $m_{G}$.

- $R_{G}(x, X)$ is the rank of the restriction of the symplectic form to the orbit $G x$.

- $R_{G}(X)=\max _{x \in X} R_{G}(x, X)$.

- $\operatorname{def}_{G}(X)$ is the defect of the action $G: X, \operatorname{def}_{G}(X):=m_{G}(X)-R_{G}(X)$.

- $G^{\circ}$ is the connected (=irreducible) component of the unit element in the algebraic group $G$.

For a linear group $G \subset \mathrm{GL}(U)$ by $G^{\text {sympl }}, G^{\text {ort }}$ we denote the image of $G$ under the natural inclusion of $\mathrm{GL}(U)$ in $\operatorname{Sp}\left(U \oplus U^{*}\right), \mathrm{SO}\left(U \oplus U^{*}\right)$ respectively.

By a small German letter we denote the Lie algebra of the algebraic group denoted by the corresponding capital Latin letter.

All objects (vector spaces, algebraic manifolds, and algebraic groups) are defined over complex numbers.

\section{Preliminary notions}

1.1. The moment map. For an algebraic group $G \subset \operatorname{Sp}(V)$ the action $G: V$ is Hamiltonian with the Hamiltonian

$$
H_{x}(v)=\frac{1}{2} \omega(x v, v), \quad \forall x \in \mathfrak{g}, \quad v \in V
$$

(see, e.g., 3, Chapter 2, Section 2.1, Example 2]). The moment map $\Phi_{H}$ for a subgroup $H \subset G$ is the composition of the moment map $\Phi_{G}$ and the restriction map $\mathfrak{g}^{*} \rightarrow \mathfrak{h}^{*}$. In the case where the algebras $\mathfrak{h}$ and $\mathfrak{g}$ are reductive (this is the case we will consider in this paper) we will identify $\mathfrak{g}^{*}$ with $\mathfrak{g}$ and $\mathfrak{h}^{*}$ with $\mathfrak{h}$ in a compatible way (e.g., using an invariant form on $\mathfrak{g}$ that is nondegenerate on $\mathfrak{h}$ ).

Let us present the main facts about the moment map that we will need later (see, e.g., [3. Chapter 2, Sections 2.4-2.6]). 
Let $X$ be a symplectic smooth affine manifold with a Hamiltonian action of a reductive group $G$. For a generic point $x \in X$ we have

$$
\mathfrak{g}_{\Phi_{G}(x)} \supset \mathfrak{g}_{x} \supset\left[\mathfrak{g}_{\Phi_{G}(x)}, \mathfrak{g}_{\Phi_{G}(x)}\right] .
$$

Furthermore,

$$
\begin{aligned}
& \operatorname{ker} d_{x} \Phi_{G}=(\mathfrak{g} x)^{\perp} \quad \forall x \in X, \\
& \operatorname{dim} \Phi_{G}(X)=m_{G}(X), \\
& \operatorname{def}_{G} X=\operatorname{dim} \pi_{G}\left(\Phi_{G}(X)\right),
\end{aligned}
$$

where $\pi_{G}: \mathfrak{g} \rightarrow \mathfrak{g} / / G$ is the factorization morphism.

Following [3] we call an action $G: X$ symplectically stable if the image of the moment map $\Phi_{G}$ contains a nonempty Zariski open set consisting of semisimple elements. In this case a generic element $x \in X$ possesses the property

$$
\operatorname{def}_{G} X=\operatorname{rk} \mathfrak{g}-\operatorname{rk} \mathfrak{g}_{x} .
$$

Proposition 1. (1) If the action $G: X$ is coisotropic, then

$$
\operatorname{dim} X \leq m_{G}(X)+\operatorname{rk} \mathfrak{g} \leq \operatorname{dim} \mathfrak{g}+\text { rk } \mathfrak{g} .
$$

(2) A symplectically stable action $G: X$ is coisotropic if and only if a generic element $x \in X$ has the property

$$
\operatorname{dim} X=m_{G}(X)+\operatorname{rk} \mathfrak{g}-\operatorname{rk} \mathfrak{g}_{x} .
$$

Proof. Part 1 follows from (CO3), (1.5), and the inequality

$$
\operatorname{dim} \pi_{G}\left(\Phi_{G}(X)\right) \leq \operatorname{dim} \mathfrak{g} / / G=\operatorname{rk} \mathfrak{g} .
$$

Part 2 follows from (CO3) and (1.6).

Proposition 2. Denote by $V_{1}, V_{2}$ the spaces of two symplectic representations of a group $G$, by $v_{1}, v_{2}$ elements in $V_{1}, V_{2}$ respectively, and by $\Phi_{1}, \Phi_{2}, \Phi$ the moment maps for the actions $G: V_{1}, G: V_{2}, G: V_{1} \oplus V_{2}$ respectively. We have

$$
\Phi_{1}\left(\alpha v_{1}\right)=\alpha^{2} \Phi\left(v_{1}\right) \quad(\alpha \in \mathbb{C}), \quad \Phi\left(v_{1}+v_{2}\right)=\Phi_{1}\left(v_{1}\right)+\Phi_{2}\left(v_{2}\right) .
$$

Hence

$$
\Phi\left(V_{1} \oplus V_{2}\right)=\Phi_{1}\left(V_{1}\right)+\Phi_{2}\left(V_{2}\right) \supset \Phi_{1}\left(V_{1}\right) .
$$

Proof. The proof follows from (1.1).

1.2. Symplectic stability of symplectic representations of reductive groups. Here we indicate those symplectic representations of reductive groups that are not symplectically stable. Theorem 1 in [3, Chapter 2, Section 2.6] claims that the action $G: V=U \oplus U^{*}$, where $U$ is a $G$-module, is always symplectically stable. To formulate a more precise statement, we recall that the weight lattice $\Lambda(U)$ of a representation $U$ is the lattice in the space $\mathfrak{t}^{*}$ (where $\mathfrak{t}$ is a Cartan subalgebra of the algebra $\mathfrak{g}$ ) generated by all highest weights of the representation $G: \mathbb{C}[U]$. Denote by $\mathfrak{a}$ the subspace $\mathfrak{t}^{*} \subset \mathfrak{g}^{*} \cong \mathfrak{g}$ generated by the lattice $\Lambda(U)$.

Proposition 3. (1) $\overline{\Phi_{G}\left(U \oplus U^{*}\right)}=\overline{\operatorname{Ad}(G) \mathfrak{a}}$.

(2) The stabilizer of a generic point under the action $G: U \oplus U^{*}$ is $\mathfrak{l}_{0}$, where $\mathfrak{l}_{0}$ is the orthogonal complement (with respect to the invariant nondegenerate symmetric form on $\mathfrak{g})$ to the ideal $\mathfrak{a}$ in the algebra $\mathfrak{z}_{\mathfrak{g}}(\mathfrak{a})$.

(3) The corank of a generic orbit of the action $G: U \oplus U^{*}$ (which, by definition, equals $2 \operatorname{dim} U-m_{G}-\operatorname{def}_{G}$ ) is equal to the complexity of the action $G: U$ (i.e., the codimension of a generic orbit of the Borel subgroup $B \subset G$ ). 
Proof. See [3, Chapter 2, Section 3, Subsections 8, 9].

Proposition 4. Let $G=G^{(1)} \times G^{(2)}$. An action $G: X$ is symplectically stable if and only if the actions $G^{(1)}: X$ and $G^{(2)}: X$ are symplectically stable.

Proof. We have

$$
\Phi_{G^{(i)}}=\operatorname{pr}_{i} \circ \Phi_{G},
$$

where $\operatorname{pr}_{i}: \mathfrak{g} \rightarrow \mathfrak{g}_{i}$ is the projection to the $i$ th summand. Now the proof follows from the fact that an element $\left(x_{1}, x_{2}\right) \in \mathfrak{g}$ is semisimple if and only if both elements $x_{1} \in \mathfrak{g}_{1}$ and $x_{2} \in \mathfrak{g}_{2}$ are semisimple.

Let $V$ be a symplectic vector space. Proposition 4 implies that a representation $G: V$ is symplectically stable provided the restrictions of $G: V$ to simple normal subgroups of $G$ are symplectically stable (every symplectic action of a torus is symplectically stable by definition). Representations of the form $G: U \oplus U^{*}$, as well as representations $G: V$ with $m_{G}(V)=\operatorname{dim} G$ are always symplectically stable (in the second case, $\pi_{G}\left(\Phi_{G}(V)\right.$ ) is dense in $\mathfrak{g} / / G$ ). Therefore, everything can be reduced to the question of which representations of simple groups $G$ that are not of the form $G: U \oplus U^{*}$ and have a nontrivial generic stationary subalgebra are symplectically stable.

It is known (see [1]) that for an irreducible representation of a simple algebraic group $G$ the inequality $m_{G}(V)<\operatorname{dim} G$ holds if and only if $\operatorname{dim} V \leq \operatorname{dim} G$. All representations of simple Lie algebras with a nontrivial generic stationary subalgebra were found in [6]. Whether or not a selfadjoint irreducible representation is symplectic can be determined using Table 3. Namely, if the number $p$ in Table 3 is odd, then the corresponding irreducible representation is symplectic, and if this number is even, or if the Lie algebra under consideration is not one of the algebras listed in the first line of this table, then the corresponding representation is orthogonal. Table 3 is taken from 4 , Chapter 4, Section 3, Exercise 13].

TABLE 3. Symplecticity identification table.

\begin{tabular}{|c|c|c|c|c|c|}
\hline $\mathfrak{g}$ & $A_{4 q+1}$ & $B_{l}, l=4 q+1,4 q+2$ & $C_{l}$ & $D_{4 q+2}$ & $E_{7}$ \\
\hline$p$ & $\Lambda_{2 q+1}$ & $\Lambda_{l}$ & $\sum_{i} \Lambda_{2 i+1}$ & $\Lambda_{4 q+1}+\Lambda_{4 q+2}$ & $\Lambda_{1}+\Lambda_{3}+\Lambda_{7}$ \\
\hline
\end{tabular}

Using results of [6] and Table 3, we can list all symplectic representations $G: V$ of simple algebraic groups $G$ such that $m_{G}(V)<\operatorname{dim} G$. These representations are presented in Tables 4 and 5. In both tables, the columns contain the following data: the algebraic linear group, the dimension of the space $V$, the maximal dimension of the orbit $m_{G}(V)$, and a generic stable subalgebra $\mathfrak{h}$ together with the restriction of the simplest representation of the algebra $\mathfrak{g}$ to $\mathfrak{h}$ (in the case under consideration this determines the subalgebra $\mathfrak{h} \subset \mathfrak{g}$ up to conjugacy).

It follows from (1.2) that for symplectic stability of a representation it is necessary that a generic stationary subalgebra be reductive. Therefore the direct sum of $(2 k+1)$ copies of the tautological representation $\operatorname{Sp}(2 l)$ with $k<l$ is not symplectically stable. Now we show that all other representations in Tables 4 and 5 are symplectically stable. Indeed, the tables show that for all these linear groups $G$, stable generic subalgebras are semisimple. Therefore, it suffices to prove the following result.

Proposition 5. Let $G$ be a reductive algebraic group and $G: Y$ a Hamiltonian action of the group $G$ on a smooth algebraic manifold $Y$. Let us assume that the stabilizer $\mathfrak{h}$ 
TABLE 4. Irreducible symplectic representations of simple groups with $\operatorname{dim} V \leq \operatorname{dim} G$.

\begin{tabular}{|c|c|c|c|c|}
\hline & $G$ & $\operatorname{dim} V$ & $m_{G}$ & $\mathfrak{h} \hookrightarrow \mathfrak{g}$ \\
\hline 1 & $\operatorname{Sp}(2 l)$ & $2 l$ & $2 l$ & $\mathfrak{s p}(2 l-1)$ \\
\hline 2 & $\wedge^{3} \mathrm{SL}(6)$ & 20 & 19 & $\mathfrak{s l}(3) \oplus \mathfrak{s l}(3)$ \\
\hline 3 & $\operatorname{Spin}(11)$ & 32 & 31 & $A_{4}\left(\pi_{1}, \pi_{4}, 0\right)$ \\
\hline 4 & $\operatorname{Spin}(13)$ & 64 & 62 & $\begin{array}{c}A_{2} \times A_{2}\left(\left(\pi_{1}, 0\right),\left(\pi_{2}, 0\right),\right. \\
\left.\left(0, \pi_{1}\right),\left(0, \pi_{2}\right), 0\right)\end{array}$ \\
\hline 5 & $C_{3}\left(\pi_{3}\right)$ & 14 & 13 & $A_{2}\left(\pi_{1}, \pi_{2}\right)$ \\
\hline 6 & $\operatorname{Spin}_{ \pm}(12)$ & 32 & 31 & $A_{5}\left(\pi_{1}, \pi_{5}\right)$ \\
\hline 7 & $E_{7}\left(\pi_{1}\right)$ & 56 & 55 & $E_{6}\left(\pi_{1}, \pi_{5}\right)$ \\
\hline
\end{tabular}

TABLE 5. Reducible symplectic representations of simple groups with $m_{G}<\operatorname{dim} G$ and $V^{G}=0$.

\begin{tabular}{|c|c|c|c|c|}
\hline & $G$ & $\operatorname{dim} V$ & $m_{G}$ & $\mathfrak{h} \hookrightarrow \mathfrak{g}$ \\
\hline 1 & $A_{5}\left(\pi_{3}, \pi_{1}, \pi_{5}\right)$ & 32 & 29 & $A_{1} \times A_{1}\left(\left(\pi_{1}, 0\right),\left(0, \pi_{1}\right)\right)$ \\
\hline 2 & $B_{5}\left(\pi_{5}, \pi_{1}, \pi_{1}\right)$ & 54 & 47 & $A_{2}\left(\pi_{1}, \pi_{2}\right)$ \\
\hline 3 & $C_{l}\left(\pi_{1}, \ldots, \pi_{1}\right)$ & $2(2 k+1) l$ & $(2 l-k)(l+1)$ & $\mathfrak{s p}(2 l-2 k-1)$ \\
\hline 4 & $C_{3}\left(\pi_{1}, \pi_{3}\right)$ & 20 & 18 & $A_{1}\left(\pi_{1}, \pi_{1}\right)$ \\
\hline 5 & $D_{6}\left(\pi_{5}, \pi_{6}\right)$ & 64 & 60 & $A_{1} \times A_{1}\left(2\left(\pi_{1}, 0\right), 2\left(0, \pi_{1}\right)\right)$ \\
\hline 6 & $D_{6}\left(\pi_{5}, \pi_{1}, \pi_{1}\right)$ & 56 & 51 & $A_{3}\left(\pi_{1}, \pi_{3}\right)$ \\
\hline 7 & $D_{6}\left(\pi_{5}, \pi_{1}, \pi_{1}, \pi_{1}, \pi_{1}\right)$ & 80 & 63 & $A_{1}\left(\pi_{1}, \pi_{1}\right)$ \\
\hline
\end{tabular}

of a generic point under the action $G: Y$ is semisimple. Then the action $G: Y$ is symplectically stable.

Proof. Let $y$ be a generic point in the manifold $Y$ and $\mathfrak{g}_{y}=\mathfrak{h}$. Let us assume that $x=\Phi_{G}(y)$ and $S=\overline{\left\{\Phi_{G}(u) \mid \mathfrak{g}_{u}=\mathfrak{h}\right\}}$. Then $S \subset \mathfrak{f}=\mathfrak{z}_{\mathfrak{g}}(\mathfrak{h})$, the submanifold $S \subset \mathfrak{f}$ is invariant under the adjoint action of the group $F=\operatorname{Int}(\mathfrak{f})$, and the set $G S$ contains an open subset in $\Phi_{G}(Y)$. Formula (1.2) implies that the algebra $\mathfrak{z}_{\mathfrak{f}}(x)$ is commutative and $\mathfrak{z}_{\mathfrak{g}}(x)=\mathfrak{z}_{\mathfrak{f}}(x) \oplus \mathfrak{h}$. According to (1.5) we have

$$
\operatorname{def}_{G}(Y)=\operatorname{dim} \Phi_{G}(Y) / / G \leq \operatorname{dim} S / / F \leq \operatorname{rk} \mathfrak{f} .
$$

On the other hand,

$$
\operatorname{def}_{G}(Y)=\operatorname{dim} \mathfrak{z}_{\mathfrak{g}}(x)-\operatorname{dim} \mathfrak{h}=\operatorname{dim} \mathfrak{z}_{\mathfrak{f}}(x) \geq \operatorname{rk} \mathfrak{f} .
$$

Combining (1.10) and (1.11), we see that $\operatorname{dim} S / / F=\operatorname{rk} \mathfrak{f}$, so that $S \subset \mathfrak{f}$ contains a dense subset consisting of semisimple elements. Therefore, $\Phi_{G}(Y) \subset \mathfrak{g}$ as well.

Therefore, we have proved the following result. 
Theorem 1. A representation $G: V$ is symplectically stable if and only if among simple normal subgroups of $G$ there are no groups $G_{1}$ of the form $C_{l}, l \in \mathbb{N}$, such that $G: V$ can be decomposed into the direct sum of the trivial representation and $2 k+1$ copies of the tautological representation of $G_{1}=\operatorname{Sp}(2 l)$ with $k<l$.

Corollary 1. The direct sum of two symplectically stable representations is symplectically stable.

Remark 1. As we have shown earlier in this subsection, a representation of a simple group $G$ is symplectically stable if and only if its generic stabilizer is reductive.

1.3. Stability of symplectic representations. Let us recall that an action of a reductive algebraic group on an affine algebraic manifold is called stable if its generic orbit is closed.

The next theorem is the main result of this subsection.

Theorem 2. Let $V$ be a symplectic vector space, $G \subset \operatorname{Sp}(V)$ a reductive algebraic group. The following conditions are equivalent.

(1) The action $G: V$ is symplectically stable.

(2) The action $G: V$ is stable.

(3) A generic stabilizer for the action $G: V$ is reductive.

Proof. (1) $\Rightarrow(2)$. Let $G: X$ be a Hamiltonian symplectically stable action of a group $G$ on a smooth algebraic manifold $X$. We can view the moment map $\Phi_{G}$ as a dominant morphism from the manifold $X$ to the manifold $Y=\overline{\Phi_{G}(X)}$. It is known that there exists an open subset $U \subset \Phi_{G}(X)$ such that the restriction of $\Phi_{G}$ to the open submanifold $\Phi_{G}^{-1}(U) \subset X$ is a smooth morphism of algebraic manifolds, i.e.,

$$
\operatorname{dim} \operatorname{ker} d_{x} \Phi_{G}=\operatorname{dim} X-\operatorname{dim} Y \quad \forall x \in \Phi_{G}^{-1}(U) .
$$

This formula and (1.3) imply that for each element $x \in \Phi_{G}^{-1}(U)$ we have $\operatorname{dim} \mathfrak{g} x=$ $m_{G}(X)$. We can assume that the set $U$ is $G$-invariant and consists of semisimple elements. Now let there exist $x \in \Phi_{G}^{-1}(U)$ such that the orbit $G x$ is not closed, and let $x_{1} \in \overline{G x} \backslash G x$. We have $\Phi_{G}\left(x_{1}\right) \in \overline{G \Phi_{G}(x)}=G \Phi_{G}(x)$ because the element $\Phi_{G}(x) \in \mathfrak{g}$ is semisimple. Therefore, $\operatorname{dim} G x_{1}=m_{G}(X)$, contradicting the choice of $x_{1}$.

$(2) \Rightarrow(3)$. See [5, Subsection 4.7].

$(3) \Rightarrow(1)$. Let a generic stabilizer for the action $G: V$ be reductive. We prove that the action $G: V$ is symplectically stable. It suffices to prove that the restriction of the action $G: V$ to each simple normal subgroup of the group $G$ is symplectically stable (see Proposition (4). Let $G_{1}$ be a simple normal subgroup of $G$. It is clear that $\mathfrak{h} \cap \mathfrak{g}_{1}$ is an ideal in $\mathfrak{h}$, so that $H$ is a reductive subgroup in $G_{1}$. The remark at the end of the previous subsection implies that the representation $G_{1}: V$ is symplectically stable.

1.4. Passing to direct summands. The main tool used in the classification of reducible coisotropic representations is the following proposition.

Proposition 6. Let $G: V_{1}$ and $G: V_{2}$ be symplectic coisotropic representations such that the representation $G: V_{1} \oplus V_{2}$ is coisotropic. Let $H$ be a generic stabilizer for the action $G: V_{1}$. Then the representations $G: V_{1}$ and $H: V_{2}$ are coisotropic. Conversely, if the representations $G: V_{1}$ and $H: V_{2}$ are coisotropic, then the property that the action $G: V_{1} \oplus V_{2}$ is coisotropic is equivalent to the equality

$$
R_{G}\left(V_{1}\right)+R_{H}\left(V_{2}\right)=R_{G}\left(V_{1} \oplus V_{2}\right) .
$$

Proof. Let us prove that the actions $G: V_{1}$ and $H: V_{2}$ are coisotropic. For an arbitrary algebraic group $G$, a symplectic representation $G: W$ is coisotropic if and only if 
$R_{G}(W)=2 m_{G}(W)-\operatorname{dim} W$, whereas in the general case, $R_{G}(W) \geq 2 m_{G}(W)-\operatorname{dim} W$. It is clear that

$$
m_{G}\left(V_{1} \oplus V_{2}\right)=m_{G}\left(V_{1}\right)+m_{H}\left(V_{2}\right) .
$$

From (1.14) and the fact that the action $G: V_{1} \oplus V_{2}$ is coisotropic, we have

$$
\begin{aligned}
R_{G}\left(V_{1} \oplus V_{2}\right) & =2 m_{G}\left(V_{1} \oplus V_{2}\right)-\operatorname{dim} V_{1}-\operatorname{dim} V_{2} \\
& =2 m_{G}\left(V_{1}\right)-\operatorname{dim} V_{1}+2 m_{H}\left(V_{2}\right)-\operatorname{dim} V_{2} \leq R_{G}\left(V_{1}\right)+R_{H}\left(V_{2}\right) .
\end{aligned}
$$

To prove the formulas

$$
R_{G}\left(V_{1}\right)=2 m_{G}\left(V_{1}\right)-\operatorname{dim} V_{1}, \quad R_{H}\left(V_{2}\right)=2 m_{H}\left(V_{2}\right)-\operatorname{dim} V_{2},
$$

which are equivalent to the fact that the actions $G: V_{1}$ and $H: V_{2}$ are coisotropic, it suffices to show that for arbitrary symplectic representations $G: V_{1}$ and $G: V_{2}$ we have

$$
R_{G}\left(V_{1} \oplus V_{2}\right) \geq R_{G}\left(V_{1}\right)+R_{H}\left(V_{2}\right) .
$$

Let vectors $v_{1} \in V_{1}, v_{2} \in V_{2}$ be such that

$$
r_{G}\left(v_{1}, V_{1}\right)=R_{G}\left(V_{1}\right), \quad r_{H}\left(v_{2}, V_{2}\right)=R_{H}\left(V_{2}\right), \quad G_{v_{1}}=H .
$$

Choose a basis $h_{1}, \ldots, h_{l} \in \mathfrak{h}$, complement it to a basis $g_{1}, \ldots, g_{m}$ in $\mathfrak{g}$, and compute the matrix $\Omega$ of the restriction of the form $\omega$ to $T_{v_{1}+t v_{2}} G\left(v_{1}+t v_{2}\right)$ in the basis $\left\{e_{i}=t h_{i} v_{2}\right.$, $\left.f_{j}=g_{j}\left(v_{1}+t v_{2}\right)\right\}$ :

$$
\begin{aligned}
& \omega\left(e_{i}, e_{j}\right)=t^{2} \omega_{2}\left(h_{i} v_{2}, h_{j} v_{2}\right), \\
& \omega\left(e_{i}, f_{j}\right)=\omega\left(t h_{i} v_{2}, g_{j}\left(t v_{2}+v_{1}\right)\right)=t^{2} \omega_{2}\left(h_{i} v_{2}, g_{j} v_{2}\right), \\
& \omega\left(f_{i}, f_{j}\right)=\omega\left(g_{i}\left(t v_{2}+v_{1}\right), g_{j}\left(t v_{2}+v_{1}\right)\right)=\omega_{1}\left(g_{i} v_{1}, g_{j} v_{1}\right)+t^{2} \omega_{2}\left(g_{i} v_{2}, g_{j} v_{2}\right) .
\end{aligned}
$$

To prove (1.16), it remains to consider the minor of the matrix $\Omega$ formed by those rows and columns where one has nonzero minors of the matrices $\left(\omega_{1}\left(g_{i} v_{1}, g_{j} v_{1}\right)\right)$ and $\left(\omega_{2}\left(h_{i} v_{2}, h_{j} v_{2}\right)\right)$, and to take the summand of this minor that has the smallest degree in $t$.

Conversely, let the actions $G: V_{1}$ and $H: V_{2}$ be coisotropic and let (1.13) hold. In this case,

$$
\begin{aligned}
2 m_{G}\left(V_{1} \oplus V_{2}\right)-R_{G}\left(V_{1} \oplus V_{2}\right) & =2 m_{G}\left(V_{1}\right)-R_{G}\left(V_{1}\right)+2 m_{H}\left(V_{2}\right)-R_{H}\left(V_{2}\right) \\
& =\operatorname{dim} V_{1}+\operatorname{dim} V_{2},
\end{aligned}
$$

which is equivalent to the fact that the action $G: V_{1} \oplus V_{2}$ is coisotropic.

The verification of whether equality (1.13) holds for arbitrary $G, V_{1}, V_{2}$ seems to be rather difficult. However, in some special cases this can be done.

Proposition 7. Let representations $G: V_{1}, V_{2}$ be symplectically stable. Then (1.13) holds.

Proof. In this case the action $G: V=V_{1} \oplus V_{2}$ is symplectically simple (this follows from Theorem 11), hence stable (this follows from Theorem 2). Let $H$ be a generic stabilizer for the action $G: V_{1}$. The action $H: V_{2}$ is stable (this follows from the theorem about étale slice, see, e.g., [5, §6]), hence symplectically stable. Therefore, for a generic vector $v \in V_{2}$ we have

$$
\begin{aligned}
R_{G}\left(V_{1}\right) & =\operatorname{dim} \mathfrak{g}-\operatorname{dim} \mathfrak{h}-\operatorname{rk} \mathfrak{g}+\operatorname{rk} \mathfrak{h}, \\
R_{H}\left(V_{2}\right) & =\operatorname{dim} \mathfrak{h}-\operatorname{dim} \mathfrak{h}_{v}-\operatorname{rk} \mathfrak{h}+\operatorname{rk} \mathfrak{h}_{v}, \\
R_{G}\left(V_{1} \oplus V_{2}\right) & =\operatorname{dim} \mathfrak{g}-\operatorname{dim} \mathfrak{h}_{v}-\operatorname{rk} \mathfrak{g}+\operatorname{rk} \mathfrak{h}_{v}=R_{G}\left(V_{1}\right)+R_{H}\left(V_{2}\right) .
\end{aligned}
$$


Proposition 8. Let $G=G_{0} \times G_{1} \times G_{2}$ be a reductive group and $V_{1}$, $V_{2}$ the spaces of symplectic representations of the group $G$ that are trivial on $G_{2}$ and $G_{1}$ respectively. Let us assume that the projections of generic stable subalgebras for the actions $G: V_{1}$ and $G: V_{2}$ to $\mathfrak{g}_{0}$ have trivial intersection. Let us assume also that the action $G: V_{1}$ is symplectically stable and coisotropic. Denote by $H_{0}$ the projection to $G_{0}$ of a generic stabilizer for the action $G: V_{1}$. The representation $G: V=V_{1} \oplus V_{2}$ is coisotropic if and only if the representation $H_{0} \times G_{2}: V_{2}$ is coisotropic.

Proof. The hypotheses of the proposition imply that $m_{G_{0}}(V)=\operatorname{dim} G_{0}$ and that a generic stabilizer for the action $G: V$ is of the form $H=H_{1} \times H_{2}$, where $H_{i}, i=1,2$, is a generic stabilizer for the action $G_{i}: V_{i}, i=1,2$, defined as the restriction of the action $G: V_{i}$ to $G_{i}$. The groups $H_{0}$ and $H_{1}$ are reductive since the representation $G_{1} \times G_{0}: V_{1}$ is symplectically stable.

Let $\left(v_{1}, v_{2}\right)$ be a generic point in the space $V=V_{1} \oplus V_{2}$. Let $\Phi_{G_{1} \times G_{0}}\left(v_{1}\right)=\left(x_{1}, x_{0}^{1}\right)$, $\Phi_{H_{0} \times G_{2}}\left(v_{2}\right)=\left(x_{0}^{2}, x_{2}\right)$, where $x_{1} \in \mathfrak{g}_{1}, x_{0}^{1} \in \mathfrak{g}_{0}, x_{0}^{2} \in \mathfrak{h}_{0}, x_{2} \in \mathfrak{g}_{2}$. Part 2 of Proposition 2 implies that $\Phi_{G}\left(v_{1}, v_{2}\right)=\left(x_{0}, x_{1}, x_{2}\right), x_{i} \in \mathfrak{g}_{i}$.

Projections to $\mathfrak{g}_{0}, \mathfrak{h}_{0}$ of generic stabilizers for the actions $G: V_{1} \oplus V_{2}, H_{0} \times G_{2}: V_{2}$ respectively are trivial. Therefore, $x_{0}^{1}$ and $x_{0}^{2}$ are regular semisimple elements in $\mathfrak{g}_{0}$ and $\mathfrak{h}_{0}$ respectively. Hence,

$$
\begin{aligned}
R_{G}(V) & =\operatorname{dim} G-\operatorname{dim} G_{\left(x_{0}, x_{1}, x_{2}\right)} \\
& =\operatorname{dim} G-\operatorname{dim} \mathfrak{z}_{\mathfrak{g}_{1}}\left(x_{1}\right)-\operatorname{rk} \mathfrak{g}_{0}-\operatorname{dim} \mathfrak{z}_{\mathfrak{g}_{2}}\left(x_{2}\right), \\
R_{H_{0} \times G_{2}}\left(V_{2}\right) & =\operatorname{dim} \mathfrak{h}_{0}+\operatorname{dim} \mathfrak{g}_{2}-\operatorname{rk} \mathfrak{h}_{0}-\operatorname{dim} \mathfrak{z}_{\mathfrak{g}_{2}}\left(x_{2}\right) .
\end{aligned}
$$

Since the representation $G_{1} \times G_{0}: V_{1}$ is symplectically stable, the elements $x_{1}$ and $x_{0}^{1}$ are semisimple. Therefore,

$$
R_{G}\left(V_{1}\right)=\operatorname{dim} \mathfrak{g}_{1}+\operatorname{dim} \mathfrak{g}_{0}-\operatorname{rk} \mathfrak{g}_{0}-\operatorname{dim} \mathfrak{z}_{\mathfrak{g}_{1}}\left(x_{1}\right)-\operatorname{dim} \mathfrak{h}_{0}+\operatorname{rk} \mathfrak{h}_{0} .
$$

Taking the sum of (1.21) and (1.22), we see that $R_{G}\left(V_{1} \oplus V_{2}\right)=R_{G}\left(V_{1}\right)+R_{H_{0} \times G_{2}}\left(V_{2}\right)$. Using Proposition 6, we complete the proof.

To determine when the intersection of one subgroup and an appropriate conjugate of another subgroup is discrete, is a rather complicated problem. The next proposition answers this question in an important special case.

Proposition 9. Let $\mathfrak{g}$ be a semisimple Lie algebra, $\mathfrak{f}, \mathfrak{h}$ subalgebras of $\mathfrak{g}$. If the algebra $\mathfrak{f}$ is commutative, its unipotent radical is at most one-dimensional, and each subalgebra conjugate to $\mathfrak{h} \subset \mathfrak{g}$ has a nontrivial intersection with $\mathfrak{f}$, then $\mathfrak{h}$ contains all simple ideals of the algebra $\mathfrak{g}$ to which $\mathfrak{f}$ is projected isomorphically.

Proof. The set of algebraic subalgebras in the Lie algebra $\mathfrak{f}$ is countable. Therefore, there exists a subalgebra $\mathfrak{f}_{1} \subset \mathfrak{f}$ contained in $\operatorname{Ad}(g) \mathfrak{h}$ for each $g \in \operatorname{Int}(\mathfrak{g})$. Therefore, $\operatorname{Ad}(G) \mathfrak{f}_{1} \subset \mathfrak{h}$, and the proof is completed.

\section{Classification of COISOtropic Representations}

Proposition [6 shows that the classification of isotropic representations can be made in several steps.

(1) Classification of irreducible coisotropic representations $G: V$. In this case we can assume that the group $G$ is semisimple because groups with nondiscrete center do not have irreducible selfadjoint locally exact representations. The case of a simple group $G$ is treated in 2.1, and of a nonsimple group, in 2.2 
(2) Classification of coisotropic representations of the form $G: U \oplus U^{*}$. Proposition 3 implies that the action $G: U \oplus U^{*}$ is coisotropic if and only if the action $G: U$ is spherical. The classification of irreducible spherical representations was obtained in [9], and the general case was treated, e.g., in [10. The results of classification are presented in 2.3 .

(3) Classification of coisotropic representations with nontrivial symplectic direct summands, at least one of them irreducible. This case is treated in 2.4

\subsection{Irreducible coisotropic representations of simple groups.}

Theorem 3. (1) Simple irreducible coisotropic linear groups $G$ with $m_{G}<\operatorname{dim} G$ are precisely those listed in Table 4 .

(2) There exists exactly one simple irreducible coisotropic group with $m_{G}=\operatorname{dim} G$. This is the group $S^{3} \mathrm{SL}(2)$.

Proof. The linear group $G=\mathrm{Sp}(2 l)$ has a dense orbit, hence is coisotropic. For other groups in Table 4 and for the group $S^{3} \mathrm{SL}(2)$, the corresponding representations are symplectically stable (by Theorem 11); hence everything is reduced to the verification of formula (1.8).

Let us prove that there are no other irreducible coisotropic representations of simple groups. According to Part 1 of Proposition 1, it suffices to prove that this theorem lists all irreducible representations of simple groups with $\operatorname{dim} V \leq \operatorname{dim} G+\operatorname{rk} G$. Since the dimension of an irreducible representation is a nondecreasing function of its highest weight (by the Weyl formula), Table 3 shows that the irreducible representations with the following highest weights have dimension greater than $\operatorname{dim} G+\operatorname{rk} G$ are as follows:

(1) $\pi_{1}+\pi_{3}, \pi_{2}+\pi_{3}, 2 \pi_{3}$ for the group $G$ of type $A_{5}$.

(2) $\pi_{l}$ for the group $G$ of type $A_{l}, l \geq 9$.

(3) $\pi_{l}+\pi_{i}, i<l$, for the group $G$ of type $B_{l}, l=2,5,6$.

(4) $\pi_{l}$ for the group $G$ of type $B_{l}, l \geq 9$.

(5) $3 \pi_{1}, \pi_{1}+\pi_{3}, \pi_{2}+\pi_{3}, 2 \pi_{3}$ for the group $G$ of type $C_{3}$.

(6) $3 \pi_{1}, \pi_{1}+\pi_{2}, \pi_{i}, 2<i \leq 4$, for the group $G$ of type $C_{l}, l>3$.

(7) $\pi_{5}+\pi_{i}, i<5,2 \pi_{5}, \pi_{5}+\pi_{6}$ for the group $G$ of type $D_{6}$.

(8) $\pi_{l-1}$ for the group $G$ of type $D_{l}, l \geq 10$.

(9) $2 \pi_{1}, \pi_{i}, i>1, i \neq 6, \pi_{1}+\pi_{6}$ for $G=E_{7}$.

This can be verified using Table 5 in the reference section in [4] and Table 2 in [2].

\subsection{Irreducible coisotropic representations of nonsimple groups.}

Theorem 4. Irreducible coisotropic representations of nonsimple groups are precisely those listed in Table 6 .

The proof of this theorem is presented later in this subsection.

Table [6 contains the following data: the linear group $G$, the dimension of the space $V$ where the group acts, the stabilizer of a generic point. All stable subalgebras except that in the case $G=\operatorname{Sp}(4) \otimes \operatorname{Spin}(7)$ are computed in [7, where this particular case was omitted. In this latter case the subalgebra $\mathfrak{h}$ is determined by the following arguments. On one hand, this subalgebra has trivial intersection with the factor $\mathfrak{s p i n}(7)$ since the stabilizer of a generic point in the sum of four copies of the spinor representation of the algebra $\mathfrak{s o}(7)$ is trivial (see [6]), i.e., $\operatorname{dim} \mathfrak{h} \leq 2$ and $\mathfrak{h}$ is conjugate to a subalgebra in $\mathfrak{t}(2)$. On the other hand, for each symplectic representation $G: V$ we have $m_{G}(V)+\operatorname{def}_{G}(V) \leq$ $\operatorname{dim} V$, and hence $\operatorname{dim} \mathfrak{h} \geq 2$.

The semisimple part of the algebra $\mathfrak{h}$ lies in the second (orthogonal) factor of the group $G$ (see [7]) in the cases $G=\operatorname{Sp}(2 m) \otimes \mathrm{SO}(n), n>2 m+2, \mathrm{SL}(2) \otimes \operatorname{Spin}(7)$, 
TABLE 6. Irreducible coisotropic representations of nonsimple groups.

\begin{tabular}{|c|c|c|c|}
\hline & $G$ & $\operatorname{dim} V$ & $\mathfrak{h}$ \\
\hline 1 & $\mathrm{Sp}(2 m) \otimes \mathrm{SO}(n)$ & $2 m n$ & $\begin{array}{c}n \geq 2 m: \mathrm{SO}(n-2 m) \times \mathfrak{t}(m) \\
2 m>n: \mathrm{Sp}(2 m-n) \times \mathfrak{t}([n / 2])\end{array}$ \\
\hline 2 & $\mathrm{Sp}(2 m) \otimes \operatorname{Spin}(7)$ & $16 m$ & $\begin{array}{c}m=1: A_{2} \times \mathfrak{t}(1) \\
m=2: \mathfrak{t}(2) \\
\end{array}$ \\
& & & $\begin{array}{c}m=3,4: 0 \\
\end{array}$ \\
\hline 3 & $\mathrm{SL}(2) \otimes \operatorname{Spin}(9)$ & 32 & $m \geq \mathfrak{s p}(2 m-8)$ \\
\hline 4 & $\mathrm{SL}(2) \otimes G_{2}\left(\pi_{1}\right)$ & 14 & $A_{2} \times \mathfrak{t}(1)$ \\
\hline 5 & $\mathrm{Sp}(4) \otimes G_{2}\left(\pi_{1}\right)$ & 28 & $A_{1} \times \mathfrak{t}(1)$ \\
\hline
\end{tabular}

$\operatorname{SL}(2) \otimes \operatorname{Spin}(9)$, and $\operatorname{SL}(2) \otimes G_{2}$, and coincides with a generic stabilizer for the action of this factor on $V$.

Table 6] shows that the number of simple factors of an irreducible coisotropic group $G$ does not exceed 3 (and equals 3 for the group $G=\mathrm{Sp}(2 m) \otimes \mathrm{SO}(4)=\mathrm{Sp}(2 m) \otimes \mathrm{SL}(2) \otimes$ $\mathrm{SL}(2)$ only).

2.2.1. Preliminary comments. Let $G=G^{(1)} \otimes \cdots \otimes G^{(k)}$ be an irreducible linear group, where the groups $G^{(i)}$ are simple. The group $G$ is symplectic if and only if among the groups $G^{(i)}$ there is an odd number of symplectic groups, and the remaining groups are orthogonal.

Therefore, if a linear group $G$ is not a group of the form $\operatorname{Sp}(2 m) \otimes \mathrm{SO}(n)$, we can embed $G$ into a group of one of the following types:

(1) $\operatorname{Sp}(2 m) \otimes G^{(2)}$, where $G^{(2)}$ is a simple irreducible orthogonal group different from $\mathrm{SO}(n)$.

(2) $G^{(1)} \otimes \mathrm{SO}(n)$, where $G^{(1)}$ is an irreducible symplectic group different from $\operatorname{Sp}(2 m)$.

(3) $\mathrm{Sp}(2 m) \otimes \mathrm{SO}\left(n_{1}\right) \otimes \mathrm{SO}\left(n_{2}\right), n_{1}, n_{2} \geq 3$.

(4) $\mathrm{Sp}\left(2 m_{1}\right) \otimes \mathrm{Sp}\left(2 m_{2}\right) \otimes \mathrm{Sp}\left(2 m_{3}\right)$, where at most one of the numbers $m_{i}$ equals 1.

To prove Theorem 4 we need to show that the linear group $\mathrm{Sp}(2 m) \otimes \mathrm{SO}(n)$ is coisotropic, that there are no coisotropic groups of the form (2), (3), and (4), and that coisotropic representations of a group of the form (1) are precisely those listed in Table 6. The proofs of these statements will be carried out in the corresponding subsubsections, while here we prove a proposition we will need in these subsections.

Proposition 10. Let $G=\mathrm{Sp}(2 m) \otimes G^{(2)}, G^{(2)} \subset \mathrm{SO}(n), 2 m>n$. The linear group $G$ is coisotropic if and only if the group $\operatorname{Sp}\left(2\left[\frac{n+1}{2}\right]\right) \otimes G^{(2)}$ is coisotropic. Similarly, if $n>2 m$, then the linear group $G^{(1)} \otimes \mathrm{SO}(n), G^{(1)} \subset \mathrm{Sp}(2 m)$ is coisotropic if and only if the linear group $G^{(1)} \otimes \mathrm{SO}(2 m)$ is coisotropic.

Proof. Let $U$ be the space of the tautological representation of $\operatorname{Sp}(2 m)$. Take a subspace $U^{\prime \prime} \subset U$ of dimension $2\left(m-\left[\frac{n+1}{2}\right]\right)$ such that the restriction of the symplectic form to $U^{\prime \prime}$ is nondegenerate. Let $U^{\prime}$ be the orthogonal complement to $U^{\prime \prime}$. The groups $\operatorname{Sp}\left(U^{\prime}\right)$ and $\operatorname{Sp}\left(U^{\prime \prime}\right)$ can be naturally embedded in $\operatorname{Sp}(2 m)$. Let $W$ be the space of the tautological representation of $\mathrm{SO}(n), v \in U^{\prime} \otimes W$ a generic vector, and $\mathfrak{m}$ the $\left(\operatorname{Sp}\left(U^{\prime}\right) \times \operatorname{Sp}\left(U^{\prime \prime}\right)\right)$ invariant complement to $\mathfrak{s p}\left(U^{\prime}\right) \oplus \mathfrak{s p}\left(U^{\prime \prime}\right)$ in $\mathfrak{s p}(U)$. One can easily notice that $\mathfrak{m} v=$ $U^{\prime \prime} \otimes W$. 
A generic element $u \in U \otimes W$ is conjugate to an element in $U^{\prime} \otimes V$. Therefore the action $G: U \otimes W$ is coisotropic if and only if for each $v \in U^{\prime} \otimes V$ the subspace $\mathfrak{g} v \subset U \otimes W$ is coisotropic. Let $\mathfrak{g}_{0}=\mathfrak{s p}\left(U^{\prime}\right) \times \mathfrak{g}^{(2)}$; this is a subalgebra of $\mathfrak{g}$. We have $\mathfrak{g} v=\mathfrak{g}_{0} v \oplus \mathfrak{m} v=\mathfrak{g}_{0} v \oplus U^{\prime \prime} \otimes W$. Therefore, the subspace $\mathfrak{g} v$ is coisotropic in $U \otimes V$ if and only if the subspace $\mathfrak{g}_{0} v$ is coisotropic in $U^{\prime} \otimes V$, and we receive the desired result.

The proof for $G^{(1)} \otimes \mathrm{SO}(n)$ is similar.

2.2.2. Cosiotropicity of $\mathrm{Sp}(2 m) \otimes \mathrm{SO}(n)$. Here we prove that the linear group $\mathrm{Sp}(2 m) \otimes$ $\mathrm{SO}(n)$ is coisotropic. By Proposition 10 it suffices to consider the cases $n=2 m$ and $n=$ $2 m-1$. Denote by $V$ the space of the representation of the linear group $\operatorname{Sp}(2 m) \otimes \operatorname{SO}(n)$.

The representation of $G=\operatorname{Sp}(2 m) \times \mathrm{SO}(2 m): V$ is symplectically stable (by Theorem 11). The stabilizer of a generic point for the action $G: V$ is an $m$-dimensional diagonalizable subalgebra $\mathfrak{t}(m)$, which has zero intersection with the subalgebras $\mathfrak{s p}(2 m), \mathfrak{s o}(2 m) \subset$ $\mathfrak{g}$. The group $G$ is coisotropic by Part 2 of Proposition 1 .

Now let $G=\mathrm{Sp}(2 m) \otimes \mathrm{SO}(2 m-1)$. The stabilizer of a generic point for the action $G: V$ is the direct product of a one-dimensional unipotent subalgebra in $\mathfrak{s p}(2 m)$ and an $(m-1)$-dimensional diagonalizable subalgebra, which, again, has zero intersection with the subalgebras $\mathfrak{s p}(2 m), \mathfrak{s o}(2 m-1) \subset \mathfrak{g}$. Therefore,

$$
\operatorname{def}_{G} V=\operatorname{dim} \pi_{G}\left(\Phi_{G}(V)\right) \geq \operatorname{dim} \pi_{G}\left(\Phi_{\mathrm{SO}(2 m-1)}(V)\right)=m-1 .
$$

On the other hand, $m_{G}(V)=\operatorname{dim} V-(m-1)$. Taking into account (2.1), we get the desired result.

2.2.3. The case $G=\operatorname{Sp}\left(2 m_{1}\right) \otimes \operatorname{Sp}\left(2 m_{2}\right) \otimes \operatorname{Sp}\left(2 m_{3}\right), m_{1} \geq m_{2} \geq m_{3}, m_{2}>1$. In this case inequality (1.7) in Part 1 of Proposition 1 can be rewritten in the form

$$
m_{1}^{2}+m_{1}+m_{2}^{2}+m_{2}+m_{3}^{2}+m_{3} \geq 4 m_{1} m_{2} m_{3} .
$$

By Proposition 10, we can assume that $m_{1} \leq 2 m_{2} m_{3}$. If we increase $m_{1}$ keeping $m_{1}+m_{2}$ fixed, the left-hand side of inequality (2.2) increases and the right-hand side decreases. The same is true for the pair of numbers $m_{1}, m_{3}$. Therefore, we can assume that $m_{1} \geq$ $2\left(m_{2}-1\right) m_{3}-1$. In this case (2.2) implies that

$$
\begin{aligned}
0 \leq & m_{1}\left(m_{1}+1-4 m_{2} m_{3}\right)+m_{2}^{2}+m_{2}+m_{3}^{2}+m_{3} \\
& \Longrightarrow\left(2\left(m_{2}-1\right) m_{3}-1\right)\left(2 m_{2} m_{3}-1\right) \leq m_{2}^{2}+m_{2}+m_{3}^{2}+m_{3} \\
& \Longleftrightarrow\left(2 m_{2} m_{3}-2 m_{3}\right)\left(2 m_{2} m_{3}-1\right)<\left(m_{2}+m_{3}\right)\left(m_{2}+m_{3}+1\right) \\
& \Longrightarrow 2 m_{2} m_{3}<m_{2}+3 m_{3} .
\end{aligned}
$$

The latter inequality is possible only when $m_{2}=2, m_{3}=1$. Therefore, $m_{1}<4$, and inequality (2.2) can be rewritten in the form

$$
m_{1}^{2}+m_{1}+8 \geq 8 m_{1} \text {. }
$$

In the case $1<m_{1}<4$ the above inequality has no solutions. Therefore, the linear group $G$ is not coisotropic by Part 1 of Proposition 1 .

2.2.4. The case $G=\mathrm{Sp}(2 m) \otimes \mathrm{SO}\left(n_{1}\right) \otimes \mathrm{SO}\left(n_{2}\right), n_{2} \geq n_{1}>2$. In this case the inequality (1.7) can be rewritten in the form

$$
2 m^{2}+2 m+\left[\frac{n_{1}^{2}}{2}\right]+\left[\frac{n_{2}^{2}}{2}\right] \geq 2 m n_{1} n_{2} .
$$

By Proposition 10 we can assume that

$$
2 m \leq n_{1} n_{2}+1, \quad n_{2} \leq 2 m n_{1} .
$$

First we consider the case

$$
2 m+1<n_{2} n_{1} .
$$


Let us show that

$$
\frac{n_{1}^{2}+n_{2}^{2}}{2} \geq 2 m^{2}+2 m
$$

Indeed, otherwise

$$
4 m^{2}+4 m>2 m n_{2} n_{1} \Longleftrightarrow 2 m+2>n_{2} n_{1},
$$

contradicting (2.6). Therefore,

$$
\left(\frac{n_{2}}{n_{1}}\right)^{2}-2 m \frac{n_{2}}{n_{1}}+1>0 \Longleftrightarrow n_{2}>n_{1}\left(m+\sqrt{m^{2}-1}\right) .
$$

Formulas (2.4), (2.5), and (2.7) imply

$$
\begin{aligned}
2 m^{2} & +2 m+\frac{n_{1}^{2}}{2}+n_{2}\left(\frac{n_{2}}{2}-2 n_{1} m\right) \geq 0 \\
& \Longrightarrow 2 m^{2}+2 m-n_{1}^{2}\left(-\frac{1}{2}+m\left(m+\sqrt{m^{2}-1}\right)\right) \geq 0
\end{aligned}
$$

which is impossible since $n_{1}>2$.

Therefore,

$$
2 m-1 \leq n_{1} n_{2} \leq 2 m+1 .
$$

Formulas (2.4), (2.5), and (2.9) imply that

$$
n_{1}^{2}+n_{2}^{2} \geq 2 m\left(2 n_{1} n_{2}-2 m-2\right) \geq\left(n_{1} n_{2}-1\right)\left(n_{1} n_{2}-3\right) .
$$

This is impossible if $n_{1}, n_{2}>2$. Therefore, (2.4) does not hold and the action $G: V$ is not coisotropic by Part 1 of Proposition 1 .

2.2.5. The case $G=G^{(1)} \otimes \mathrm{SO}(n)$, where $G^{(1)}$ is a simple irreducible proper subgroup of $\mathrm{Sp}(2 m), n>2$. We can assume that $n \leq 2 m$ (Proposition 10). Inequality (1.7) can be rewritten in the form

$$
\operatorname{dim} \mathfrak{g}^{(1)}+\operatorname{rk} \mathfrak{g}^{(1)} \geq 2 m n-\left[\frac{n^{2}}{2}\right],
$$

whence $\operatorname{dim} \mathfrak{g}^{(1)}+r k \mathfrak{g}^{(1)} \geq 3 m\left(m>1\right.$, otherwise there is no group $G^{(1)}$ with the required properties), i.e. (see the proof of Theorem 3), $\mathfrak{g}^{(1)}$ is one of the subalgebras in Table 4. However, for $2<n \leq 2 m$ neither of these subalgebras satisfies (2.10). Hence the representation $G: V$ is not coisotropic by Part 1 of Proposition 1 .

2.2.6. Coisotropic subgroups of the form $\operatorname{Sp}(2 m) \otimes G^{(2)}$, where $G^{(2)}$ is a simple proper subgroup of the group $\mathrm{SO}(n)$. One can easily see that the existence of such $G^{(2)}$ implies that $n>4$. By Proposition 10 we can assume that $2 m \leq n+1$. Inequality (1.7) can be rewritten in the form

$$
\operatorname{dim} \mathfrak{g}^{(2)}+\operatorname{rk} \mathfrak{g}^{(2)} \geq m(2 n-2 m-2) .
$$

The right-hand side of (2.11) is greater than $n$ since $n>4$. Using a result in [1] and Table 3. we can list all orthogonal representations of simple groups $G^{(2)}: W$ with $\operatorname{dim} W<$ $\operatorname{dim} G^{(2)}$.

Direct verification shows that inequality (2.11) holds for the linear groups $\operatorname{Sp}(2 m) \otimes$ $G^{(2)}$ listed in Table 6 and also for the groups $\mathrm{SL}(2) \otimes F_{4}\left(\pi_{1}\right)$ and $\mathrm{SL}(2) \otimes C_{3}\left(\pi_{2}\right)$. Let us note that by Theorem 1 in all these cases the representation $\operatorname{Sp}(2 m) \otimes G^{(2)}: V$ is symplectically stable. Therefore, the verification of coisotropicity reduces to the verification of (1.8). For the group $\mathrm{SL}(2) \otimes F_{4}\left(\pi_{1}\right)$ a generic stabilizer is isomorphic to $A_{2}$, and for $\mathrm{SL}(2) \otimes C_{3}\left(\pi_{2}\right)$, to $\mathfrak{t}(1)$ (the latter case is missing in [7]; the form of the stabilizer was communicated to the author by E. B. Vinberg). 
TABLE 7. Irreducible orthogonal linear simple groups $G^{(2)} \varsubsetneqq \operatorname{SO}(n)$ with $n<\operatorname{dim} G^{(2)}$.

\begin{tabular}{|c|c|c|}
\hline & $G^{(2)}$ & $\operatorname{dim} W$ \\
\hline 1 & $\operatorname{Spin}(7)=B_{3}\left(\pi_{3}\right)$ & 8 \\
\hline 2 & $\operatorname{Spin}(9)=B_{4}\left(\pi_{4}\right)$ & 16 \\
\hline 3 & $C_{l}\left(\pi_{2}\right), l>2$ & $2 l^{2}-l-1$ \\
\hline 4 & $G_{2}\left(\pi_{1}\right)$ & 7 \\
\hline 5 & $F_{4}\left(\pi_{1}\right)$ & 26 \\
\hline
\end{tabular}

2.3. Spherical representations. As we mentioned at the beginning of this section, for a reductive group $G$ the representation $G: U \oplus U^{*}$ is coisotropic if and only if $G: U$ is a spherical representation, i.e., there exists an open orbit of the Borel subgroup $B \subset G$ in $U$, or, equivalently, each irreducible representation of the group $G$ occurs in $\mathbb{C}[U]$ at most once.

Since the algebra $\mathbb{C}[U]$ is factorial, there exist polynomials $f_{1}, \ldots, f_{m} \in \mathbb{C}[U]^{(B)}$ such that each highest vector of an irreducible subrepresentation of $\mathbb{C}[U]$ is proportional to $f_{1}^{n_{1}} \ldots f_{m}^{n_{m}}$. The weights of the polynomials $f_{1}, \ldots, f_{m}$ are called fundamental weights of the representation $G: U$. The number $m$ is called the rank of $G: U$; this number coincides with the codimension of a generic orbit of the maximal unipotent subgroup of the group $G$.

Now we present results of the classification of spherical representations (see [9] for irreducible representations and [10] in the general case). A spherical representation $G: U$ is called saturated if the linear group $G$ contains the torus $\mathbb{C}^{* m}=Z_{\mathrm{GL}(U)}(G)^{\circ}$. A spherical representation $G: U$ is called decomposable if $G$ can be represented in the form $G_{1} \oplus G_{2}$ and indecomposable otherwise.

Table 8 shows classification results for irreducible saturated spherical representations, and Table 9, for reducible indecomposable saturated spherical representations. Both tables contain the following data: the commutator subgroup $G^{\prime}$ of the group $G$ for which the representation $G: U$ is spherical and saturated, the restriction of fundamental weights to $G^{\prime}$, and (in parentheses) the bidegree of the corresponding highest vectors. In addition, Table 8 contains the subalgebra $\mathfrak{l}_{0} \subset \mathfrak{g}$ (see Proposition 3). The data is taken from [8]. The notation for the weights is that introduced in [4]. If $n>\operatorname{rkg}$ or $n<1$, we assume that $\pi_{n}=0$.

Let us note that all indecomposable spherical representations have at most two components.

The classification of nonsaturated spherical representations is given by the following result (see [10, Theorem 2.6]).

Proposition 11. Let $G \subset \mathrm{GL}(U)$ be a reductive linear group, $U=U_{1} \oplus \cdots \oplus U_{s}$ the decomposition of $U$ into irreducible (with respect to $G$ ) components, $\widetilde{G}$ the group generated by $G$ and the torus $\mathbb{C}^{* s}$ centralizing $G$ with the natural action $\mathbb{C}^{* s}: U$. Next, let $T \subset \widetilde{T}$ be the maximal tori of the groups $G$ and $\widetilde{G}, S$ the lattice of the characters of $\widetilde{T}$ taking the value 1 on $T$, and $\Lambda(U)$ the lattice generated by the highest weights of the representation $\widetilde{G}: \mathbb{C}[U]$. Let us assume that $\widetilde{G}: U$ is a spherical representation. Then the action $G: U$ is spherical if and only if $S \cap \Lambda(U)=\{0\}$. 
TABLE 8. Irreducible groups $G=G^{\prime} \otimes \mathbb{C}^{*}$ such that the representation $G: U$ is spherical.

\begin{tabular}{|c|c|c|}
\hline$G^{\prime}$ & $\lambda_{i}\left\{\operatorname{deg} f_{i}\right\}$ & $\mathfrak{l}_{0}$ \\
\hline $\mathrm{SL}(n)$ & $\pi_{1}\{1\}$ & $\mathfrak{g l}(n-1)$ \\
\hline $\operatorname{Sp}(2 n)$ & $\pi_{1}\{1\}$ & $\mathfrak{s p}(2 n-2) \times \mathfrak{t}(1)$ \\
\hline $\mathrm{SO}(n), n>2$ & $\pi_{1}\{1\}, 0\{2\}$ & $\mathfrak{s o}(n-2)$ \\
\hline$S^{2} \mathrm{SL}(n), n>1$ & $2 \pi_{i}\{i\}, i=1, \ldots, n$ & 0 \\
\hline$\bigwedge^{2} \mathrm{SL}(n), n>4$ & $\pi_{2 i}\{i\}, i=1, \ldots,[n / 2]$ & {$[n / 2] \mathfrak{s l}(2) \times 2\{n / 2\} \mathfrak{t}(1)$} \\
\hline $\operatorname{Spin}(7)$ & $\pi_{3}\{1\}, 0\{2\}$ & $A_{2}$ \\
\hline $\operatorname{Spin}(9)$ & $\pi_{4}\{1\}, 0\{2\}, \pi_{1}\{2\}$ & $A_{2}$ \\
\hline $\operatorname{Spin}_{ \pm}(10)$ & $\pi_{4}\{1\}, \pi_{1}\{2\}$ & $A_{3} \times \mathfrak{t}(1)$ \\
\hline$E_{6}\left(\pi_{1}\right)$ & $\pi_{1}\{1\}, \pi_{5}\{2\}, 0\{3\}$ & $D_{4}$ \\
\hline$G_{2}\left(\pi_{1}\right)$ & $\pi_{1}\{1\}, 0\{2\}$ & $A_{1}$ \\
\hline $\begin{array}{l}\mathrm{SL}(n) \otimes \mathrm{SL}(m) \\
n \geq m \geq 2, n \neq 2\end{array}$ & $\left(\pi_{i}, \pi_{i}\right)\{i\}, i=1, \ldots, m$ & $\mathfrak{s l}(n-m) \times \mathfrak{t}(m)$ \\
\hline $\begin{array}{c}\mathrm{Sp}(2 n) \otimes \mathrm{SL}(2) \\
n>1\end{array}$ & $\begin{array}{c}\left(\pi_{1}, \pi_{1}\right)\{1\}, 0\{2\} \\
\left(\pi_{2}, 0\right)\{2\}\end{array}$ & $\mathfrak{s p}(2 n-4) \times \mathfrak{t}(1)$ \\
\hline $\begin{array}{c}\mathrm{Sp}(2 n) \otimes \mathrm{SL}(3) \\
n>2\end{array}$ & $\begin{array}{l}\left(\pi_{1}, \pi_{1}\right)\{1\},\left(0, \pi_{2}\right)\{2\}, \\
\left(\pi_{2}, \pi_{2}\right)\{2\},\left(\pi_{1}, 0\right)\{3\}, \\
\left(\pi_{3}, 0\right)\{3\},\left(\pi_{2}, \pi_{1}\right)\{4\}\end{array}$ & $\mathfrak{s p}(2 n-6)$ \\
\hline $\mathrm{Sp}(4) \otimes \mathrm{SL}(3)$ & $\begin{array}{c}\left(\pi_{1}, \pi_{1}\right)\{1\},\left(0, \pi_{2}\right)\{2\} \\
\left(\pi_{2}, \pi_{2}\right)\{2\},\left(\pi_{1}, 0\right)\{3\} \\
\left(\pi_{2}, \pi_{1}\right)\{4\}\end{array}$ & 0 \\
\hline $\begin{array}{c}\mathrm{SL}(n) \otimes \mathrm{Sp}(4) \\
n \geq 4\end{array}$ & $\begin{array}{c}\left(\pi_{1}, \pi_{1}\right)\{1\},\left(\pi_{2}, \pi_{2}\right)\{2\} \\
\left(\pi_{2}, 0\right)\{2\},\left(\pi_{3}, \pi_{1}\right)\{3\} \\
\left(\pi_{4}, 0\right)\{4\},\left(\pi_{1}+\pi_{3}, \pi_{2}\right)\{4\}\end{array}$ & $\mathfrak{g l}(n-4)$ \\
\hline
\end{tabular}

This proposition implies, in particular, that the semisimple linear groups with spherical representations are the groups $\operatorname{SL}(n), \bigwedge^{2} \mathrm{SL}(2 m+1), \operatorname{Sp}(2 n), \operatorname{Spin}_{ \pm}(10), \operatorname{SL}(n) \otimes \operatorname{SL}(m)$, $n>m$, and $\operatorname{SL}(n) \otimes \operatorname{Sp}(4), n>4$.

2.4. Reducible coisotropic representations. In this subsection we classify reducible spherical representations $G: V$ with $V \neq U \oplus U^{*}$. First let us give a few definitions.

Let $V_{1}, V_{2}$ be symplectic vector spaces. The space $V=V_{1} \oplus V_{2}$ can be endowed with a natural symplectic form. A linear group $G \subset \operatorname{Sp}(V)$ is called decomposable if $G=G_{1} \oplus G_{2}$ for some linear groups $G_{1} \subset \operatorname{Sp}\left(V_{1}\right), G_{2} \subset \operatorname{Sp}\left(V_{2}\right)$. Otherwise the group $G$ is called indecomposable. The same terminology is used for representations. It is clear that if $G_{1}$ and $G_{2}$ are coisotropic linear groups, then the group $G_{1} \oplus G_{2}$ is also coisotropic, and vice versa. Therefore, it suffices to classify only indecomposable coisotropic representations. 
TABLE 9. Irreducible saturated spherical representations $G: U$.

\begin{tabular}{|c|c|}
\hline$G^{\prime}$ & $\lambda_{i}\left\{\operatorname{deg} f_{i}\right\}$ \\
\hline$A_{n}\left(\pi_{1}, \pi_{1}\right)$ & $\pi_{1}\{1,0\}, \pi_{1}\{0,1\}, \pi_{2}\{1,1\}$ \\
\hline$A_{n}\left(\pi_{1}, \pi_{n}\right), n>1$ & $\pi_{1}\{1,0\}, \pi_{n}\{0,1\}, 0\{1,1\}$ \\
\hline$A_{n}\left(\pi_{1}, \pi_{2}\right), n>2$ & $\pi_{i}\{2\{i / 2\},[i / 2]\}, i=1, \ldots, n+1$ \\
\hline$A_{n}\left(\pi_{n}, \pi_{2}\right), n>3$ & $\begin{array}{c}\pi_{2 i}\{0, i\}, i=1, \ldots,[(n+1) / 2] \\
\pi_{2 i-1}\{1, i\}, i=1, \ldots,[n / 2]\end{array}$ \\
\hline$C_{n}\left(\pi_{1}, \pi_{1}\right), n>1$ & $\pi_{1}\{0,1\}, \pi_{1}\{1,0\}, \pi_{2}\{1,1\}, 0\{1,1\}$ \\
\hline$D_{4}\left(\pi_{1}, \pi_{3}\right)$ & $\pi_{1}\{1,0\}, \pi_{3}\{0,1\}, 0\{2,0\}, 0\{0,2\}, \pi_{4}\{1,1\}$ \\
\hline $\begin{array}{c}A_{n} \times A_{m}\left(\left(\pi_{1}, 0\right),\right. \\
\left.\left(\pi_{1}, \pi_{1}\right)\right)\end{array}$ & $\begin{array}{r}\left(\pi_{i}, \pi_{i}\right)\{0, i\}, i=1, \ldots, \min \{n, m\}+1 \\
\left(\pi_{i+1}, \pi_{i}\right)\{1, i\}, i=0, \ldots, \min \{n, m+1\}\end{array}$ \\
\hline $\begin{array}{c}A_{n} \times A_{m}\left(\left(\pi_{n}, 0\right),\right. \\
\left.\left(\pi_{1}, \pi_{1}\right)\right), n>1\end{array}$ & $\begin{array}{c}\left(\pi_{i}, \pi_{i}\right)\{0, i\}, i=1, \ldots, \min \{n, m\}+1 \\
\left(\pi_{n}, 0\right)\{1,0\},\left(\pi_{i-1}, \pi_{i}\right)\{1, i\} \\
i=1, \ldots, \min \{n-1, m\}+1\end{array}$ \\
\hline $\begin{array}{c}A_{1} \times C_{n}\left(\left(\pi_{1}, 0\right)\right. \\
\left.\left(\pi_{1}, \pi_{1}\right)\right), n>1\end{array}$ & $\begin{array}{c}\left(\pi_{1}, 0\right)\{1,0\},\left(\pi_{1}, \pi_{1}\right)\{0,1\},\left(0, \pi_{1}\right)\{1,1\} \\
\left(0, \pi_{2}\right)\{0,2\}, 0\{0,2\}\end{array}$ \\
\hline $\begin{array}{c}A_{n} \times A_{1} \times A_{m} \\
\quad\left(\left(\pi_{1}, \pi_{1}, 0\right),\right. \\
\left.\left(0, \pi_{1}, \pi_{1}\right)\right)\end{array}$ & $\begin{array}{c}\left(\pi_{1}, \pi_{1}, 0\right)\{1,0\},\left(0, \pi_{1}, \pi_{1}\right)\{0,1\} \\
\left(\pi_{2}, 0,0\right)\{2,0\},\left(0,0, \pi_{2}\right)\{0,2\} \\
\left(\pi_{1}, 0, \pi_{1}\right)\{1,1\}\end{array}$ \\
\hline $\begin{array}{c}C_{n} \times A_{1} \times A_{m} \\
\left(\left(\pi_{1}, \pi_{1}, 0\right)\right. \\
\left.\left(0, \pi_{1}, \pi_{1}\right)\right), n>1\end{array}$ & $\begin{array}{c}\left(\pi_{1}, \pi_{1}, 0\right)\{1,0\},\left(0, \pi_{1}, \pi_{1}\right)\{0,1\} \\
\left(\pi_{2}, 0,0\right)\{2,0\},\left(0,0, \pi_{2}\right)\{0,2\}, \\
\left(\pi_{1}, 0, \pi_{1}\right)\{1,1\}, 0\{2,0\}\end{array}$ \\
\hline $\begin{array}{c}C_{n} \times A_{1} \times C_{m} \\
\left(\left(\pi_{1}, \pi_{1}, 0\right),\right. \\
\left.\left(0, \pi_{1}, \pi_{1}\right)\right), n, m>1\end{array}$ & $\begin{array}{c}\left(\pi_{1}, \pi_{1}, 0\right)\{1,0\},\left(0, \pi_{1}, \pi_{1}\right)\{0,1\} \\
\left(\pi_{2}, 0,0\right)\{2,0\},\left(0,0, \pi_{2}\right)\{0,2\}, \\
\left(\pi_{1}, 0, \pi_{1}\right)\{1,1\}, 0\{2,0\}, 0\{0,2\}\end{array}$ \\
\hline
\end{tabular}

A linear group $G \subset \operatorname{Sp}(V)$ (or the corresponding representation) is called saturated if $Z_{\mathrm{Sp}(V)}(G)^{\circ}=Z(G)$ (i.e., if $G$ has the maximal center for a given semisimple part $G^{\prime}$ ). For a given linear group $G \subset \operatorname{Sp}(V)$ there exists a saturated linear group $\widetilde{G} \subset \operatorname{Sp}(V)$ such that $(\widetilde{G}, \widetilde{G}) \subset G \subset \widetilde{G}$; moreover, the group $\widetilde{G}$ is determined uniquely up to conjugation by an element in $Z_{\mathrm{Sp}(V)}(G)$. A linear symplectic group $\widetilde{G}$ is called the saturation of the group $G$. Similarly, the representation $\widetilde{G}: V$ is called the saturation of the representation $G: V$.

Let us note that this terminology agrees with the terminology for spherical representations in the following sense. A symplectic coisotropic representation $G: U \oplus U^{*}$ is decomposable (saturated) if and only if the spherical representation $G: U$ possesses the same property. 
The following proposition provides a criterion that a group with decomposable saturation is coisotropic.

Proposition 12. Let $G$ be a reductive subgroup of the group $\operatorname{Sp}(V), \widetilde{G}=\widetilde{G}_{1} \oplus \widetilde{G}_{2}$, where $\widetilde{G}_{i} \subset \operatorname{Sp}\left(V_{i}\right), i=1,2, V=V_{1} \oplus V_{2}$ its saturation. Denote by $T_{1}$ the projection of a generic stabilizer for the action $G: V_{1}$ to the central torus of the group $G$, and by $G_{1}$ the image of $G$ under the representation in the space $V_{1}$. We assume that the linear groups $G_{1}$ and $\widetilde{G}_{2}$ are coisotropic. The group $G$ is coisotropic if and only if the representation $H=\left(\widetilde{G}_{2}, \widetilde{G}_{2}\right) T_{1}: V_{2}$ is coisotropic.

Proof. The action $H: V_{2}$ coincides with the action on $V_{2}$ of a generic stabilizer for the action of $G: V_{1}$. Furthermore, $R_{H}\left(V_{2}\right)=R_{\widetilde{G}_{2}}\left(V_{2}\right)$, whence the commutator subgroups of $H$ and $\widetilde{G}_{2}$ coincide. Now everything follows from Proposition 6 .

Below we will consider only those coisotropic groups $G$ whose saturation is indecomposable. One can easily see that this is equivalent to the condition that the commutator subgroup of the group $G$ is indecomposable (as a symplectic linear group).

Proposition 13. Let $\widehat{G}=\operatorname{SL}(2) \times \operatorname{SL}(2) \times G_{0}$ and let $\rho$ be a coisotropic locally exact representation of the group $\widehat{G}$. Let us consider the subgroup $G=\mathrm{SL}(2) \times G_{0}$, where the factor $\mathrm{SL}(2) \subset G$ is diagonally embedded in $\mathrm{SL}(2) \times \mathrm{SL}(2) \subset \widehat{G}$. Let $\widehat{H}$ (respectively $H$ ) be a generic stabilizer for the representation $\rho$ (respectively the restriction of $\rho$ to $G$ ). Then $H^{\circ} \subset G_{0}$, and the representation $\left.\rho\right|_{G}$ is coisotropic if and only if $\operatorname{codim}_{\widetilde{H}} H=2$.

Proof. Tables 4, 6, and 8 show that the projection of the group $\widehat{H}$ to each of two copies of $\operatorname{SL}(2) \subset \widehat{G}$ is of dimension at most 1. Now the inclusion $H^{\circ} \subset G_{0}$ follows from Proposition 9. Let $V$ be the space of the representation $\rho$, and $\widehat{\Phi}, \Phi$ the moment maps for the actions $\widehat{G}: V, G: V$ respectively. If $\widehat{\Phi}(v)=\left(x_{1}, x_{2}, x_{0}\right)$, then $\Phi(v)=\left(x_{1}+x_{2}, x_{0}\right)$. For a generic point $v \in V$ we have $x_{1}, x_{2} \neq 0$, and hence

$$
\begin{aligned}
& \widehat{g}_{\widehat{\Phi}(v)}=\mathbb{C}^{2} \oplus \mathfrak{z}_{\mathfrak{g}_{0}}\left(x_{0}\right), \\
& \mathfrak{g}_{\Phi(v)}=\mathbb{C} \oplus \mathfrak{z}_{\mathfrak{g}_{0}}\left(x_{0}\right)
\end{aligned}
$$

(here by equalities we mean isomorphisms of the corresponding vector spaces). Since the action $G: V$ is coisotropic if and only if

$$
\operatorname{dim} \mathfrak{g}-2 \operatorname{dim} \mathfrak{g}_{v}+\operatorname{dim} \mathfrak{g}_{\Phi(v)}=\operatorname{dim} V
$$

for a generic point $v \in V$, the desired result can be obtained by direct computation.

Remark 2. This proposition implies that the restriction of a representation of the group $\mathrm{SL}(2)^{3} \times G_{0}$ to $\mathrm{SL}(2) \times G_{0}$ (where $\mathrm{SL}(2)$ is embedded in $\mathrm{SL}(2)^{3}$ diagonally) cannot be coisotropic.

Representations $G: V$ of the form indicated in Proposition 13 are called $A_{1}$-glued and all other representations, $A_{1}$-nonglued. The latter condition is equivalent to the fact that each normal subgroup of the group $G$ that is locally isomorphic to $\mathrm{SL}(2)$ acts nontrivially on exactly one minimal symplectic direct summand of the representation $G: V$ (we assume that representations are locally exact). Since $A_{1}$-glued representations can be easily obtained from $A_{1}$-nonglued, below we will consider only the latter.

To simplify the notation, by basic reducible coisotropic representations we will mean $A_{1}$-nonglued indecomposable saturated reducible coisotropic representations $G: V$ such that there is no $G$-module $U$ with $V=U \oplus U^{*}$ (as $G$-modules). Similarly one can define basic reducible coisotropic linear groups. 
TABLE 10. Basic reducible coisotropic groups.

\begin{tabular}{|c|c|c|}
\hline & $G^{\prime}$ & $\mathfrak{h}$ \\
\hline 1 & $A_{5}\left(\pi_{3}, \pi_{1}, \pi_{5}\right)$ & $(\mathfrak{s l}(2) \oplus \mathfrak{s l}(2)) \times \mathfrak{t}(1)$ \\
\hline 2 & $C_{l}\left(\pi_{1}, \pi_{1}, \pi_{1}\right)$ & $\mathfrak{s p}(2 l-3)$ \\
\hline 3 & $C_{3}\left(\pi_{3}, \pi_{1}, \pi_{1}\right)$ & 0 \\
\hline 4 & $B_{2}\left(\pi_{1}, \pi_{1}, \pi_{2}\right)$ & 0 \\
\hline 5 & $D_{6}\left(\pi_{5}, \pi_{1}, \pi_{1}\right)$ & $A_{3}\left(\pi_{1}, \pi_{3}\right)$ \\
\hline 6 & $\operatorname{Spin}(12)$ & $A_{1} \times A_{1}\left(2\left(\pi_{1}, 0\right), 2\left(0, \pi_{1}\right)\right)$ \\
\hline 7 & $\bigwedge^{3} \operatorname{Sp}(6)$ & $A_{1}\left(\pi_{1}, \pi_{1}\right)$ \\
\hline 8 & $(\mathrm{SL}(2) \oplus \mathrm{SL}(2)) \otimes \mathrm{SO}(n), n>4$ & $\mathfrak{s o}(n-4)$ \\
\hline 9 & $\begin{array}{c}\mathrm{Sp}(2 m)+{ }_{\mathrm{Sp}(2 m)} \mathrm{Sp}(2 m) \otimes \mathrm{SO}(n) \\
m>1, n>2\end{array}$ & $\begin{array}{c}2 m>n: \mathfrak{s p}(2 m-n-1) \\
2 m \leq n: \mathfrak{s o}(n-2 m)\end{array}$ \\
\hline 10 & $\operatorname{Sp}(4)+{ }_{\operatorname{Sp}(4)} \operatorname{Sp}(4) \otimes \operatorname{Spin}(7)$ & 0 \\
\hline 11 & $(\mathrm{SL}(2) \oplus \mathrm{SL}(2)) \otimes \operatorname{Spin}(7)$ & 0 \\
\hline 12 & $\mathrm{SL}(2) \otimes \mathrm{SO}(11)+\mathrm{SO}(11) \mathrm{Spin}(11)$ & $\mathfrak{s l}(3)^{\text {ort }}$ \\
\hline 13 & $\begin{array}{c}\mathbf{S p}(\mathbf{2 m}) \otimes \mathbf{S O}(12)+\mathrm{SO}(12) \operatorname{Spin}_{ \pm}(12) \\
m=1,2\end{array}$ & $\begin{array}{c}m=1: \mathfrak{s l}(4)^{\text {ort }} \times \mathfrak{t}(1) \\
\quad m=2: \mathfrak{s l}(2)^{\text {ort }}\end{array}$ \\
\hline 14 & $\mathrm{Sp}(4)+\mathrm{Sp}(4) \mathrm{SO}(5) \otimes \mathbf{S p}(\mathbf{2 m})$ & $\begin{array}{c}m=1: \mathfrak{t}(1) \\
m>1: \mathfrak{s p}(2 m-5)\end{array}$ \\
\hline 15 & $\mathrm{SL}(2) \otimes \mathrm{SO}(7)+\mathrm{SO}(7) \operatorname{Spin}(7) \otimes \mathbf{S L}(\mathbf{2})$ & $\mathfrak{t}(1)$ \\
\hline 16 & $\mathbf{S L}(\mathbf{2}) \otimes \mathbf{S O}(8)+{ }_{\mathrm{SO}(8)} \operatorname{Spin}_{+}(8) \otimes \mathbf{S L}(\mathbf{2})$ & $A_{1} \times \mathfrak{t}(2)$ \\
\hline 17 & $\mathrm{SL}(2) \otimes \mathrm{SO}(8)+\mathrm{SO}(8) \operatorname{Spin}_{+}(8) \otimes \mathrm{Sp}(4)$ & 0 \\
\hline 18 & $\bigwedge^{3} \mathrm{SL}(6)+\mathrm{SL}(6)(\mathrm{SL}(6) \otimes \mathrm{SL}(2))^{\mathrm{sympl}}$ & $\mathfrak{t}(1)$ \\
\hline 19 & $\mathrm{SL}(2) \otimes \mathrm{SO}(5)+{ }_{\mathrm{SO}(5)} \mathrm{Sp}(4)^{\mathrm{sympl}}$ & 0 \\
\hline 20 & $\mathrm{SL}(\mathbf{2}) \otimes \mathrm{SO}(6)+\mathrm{SL}(4) \mathrm{SL}(4)^{\mathrm{sympl}}$ & $\mathfrak{t}(2)$ \\
\hline 21 & $\mathrm{SL}(2) \otimes \mathrm{SO}(7)+{ }_{\mathrm{SO}(7)} \operatorname{Spin}(7)^{\mathrm{sympl}}$ & 0 \\
\hline 22 & $\mathbf{S L}(\mathbf{2}) \otimes \mathrm{SO}(8)+{ }_{\mathrm{SO}(8)} \operatorname{Spin}_{ \pm}(8)^{\text {sympl }}$ & $A_{1} \times \mathfrak{t}(1)$ \\
\hline 23 & $\mathbf{S L}(\mathbf{2}) \otimes \mathrm{SO}(10)+\mathrm{SO}(10) \operatorname{Spin}_{ \pm}(10)^{\mathrm{sympl}}$ & $A_{1} \times \mathfrak{t}(1)$ \\
\hline
\end{tabular}

Theorem 5. Basic reducible coisotropic representations are precisely those listed in Table 10 (the column $G^{\prime}$ contains the commutator subgroup of $G$ ). Nonsaturated coisotropic groups whose saturation is one of the groups in Table 10 are just the groups $G^{\prime}$ with numbers $1,18,20$. In these three cases a generic stabilizer is a generic reductive subalgebra in $\mathfrak{h}$ of codimension 1 ; moreover, in the case of 20 its projection to $\mathfrak{s l}(2)$ is nontrivial.

Let us clarify the notation used in Table 10 .

The notation $G_{1}+G_{0} G_{2}$ means the following. Let $G_{1} \subset \operatorname{Sp}\left(U_{1}\right), G_{2} \subset \operatorname{Sp}\left(U_{2}\right)$ be reductive linear groups with Lie algebras $\mathfrak{g}_{1}, \mathfrak{g}_{2}$, and let $\mathfrak{g}_{0}$ be a Lie algebra that is embedded as an ideal in both Lie algebras $\mathfrak{g}_{1}$ and $\mathfrak{g}_{2}$, i.e., $\mathfrak{g}_{i}=\mathfrak{g}_{0} \times \mathfrak{g}_{i}^{0}$ (here $\mathfrak{g}_{1}^{0}$ and $\mathfrak{g}_{2}^{0}$ 
are some reductive Lie algebras). Consider the following linear Lie algebra:

$$
\mathfrak{f}=\left\{\left(x_{1}, x_{0}, x_{0}, x_{2}\right) \in \mathfrak{g}_{1} \oplus \mathfrak{g}_{2} \mid x_{i} \in \mathfrak{g}_{i}^{0}, x_{0} \in \mathfrak{g}_{0}\right\} .
$$

By $G_{1}+G_{0} G_{2}$ we denote the connected subgroup of the group $\operatorname{Sp}\left(U_{1} \oplus U_{2}\right)$ with Lie algebra $\mathfrak{f}$. For each of the groups listed in Table 10, the embedding $\mathfrak{g}_{0} \hookrightarrow \mathfrak{g}_{i}$ is clear from the context.

The boldface factor SL(2) in Table 10 means that the projector of a generic stabilizer to this factor is nontrivial (in Case 12 the projection of a generic stabilizer to the normal subgroup $\mathrm{SL}(2) \times \mathrm{SL}(2) \subset G$ is two-dimensional). The boldface factor of the form $\mathrm{Sp}(2 m)$ means that the above property holds in the case $m=1$.

Theorem 5 will be proved in subsequent subsections. In 2.4.1 we consider the case where the commutator subgroup $G^{\prime}=(G, G)$ is simple. Then we consider a coisotropic group with nonsimple commutator subgroup. In 2.4 .2 we find $A_{1}$-nonglued reducible coisotropic subgroups of the form $G=G^{(1)} \otimes G^{(2)}$, where $G^{(1)} \subset \mathrm{Sp}(2 m), G^{(2)} \subset \mathrm{SO}(n)$. In 2.4.3 we find those basic coisotropic representations that split into a direct sum of two irreducible symplectic representations, and in 2.4.4 those that split into a direct sum of one irreducible symplectic representation and two dual spherical representations. Finally in 2.4.5 we prove that basic reducible coisotropic representations cannot split into a direct sum of three nontrivial symplectic representations.

Finally, let us prove a statement that generalizes Proposition 10.

Proposition 14. Let $G$ be a reductive group, $\rho_{1}, \ldots, \rho_{k}$ orthogonal representations of $G$ with dimensions $p_{1}, \ldots, p_{k}, \rho_{1}^{\prime}, \ldots, \rho_{s}^{\prime}$ symplectic representations of dimensions $p_{1}^{\prime}, \ldots$, $p_{s}^{\prime}$. By $s p(2 m)$, so(n) we denote tautological representations of the groups $\operatorname{Sp}(2 m)$ and $\mathrm{SO}(n)$, respectively. The representation

$$
\bigoplus_{i=1}^{k} s p\left(2 m_{i}\right) \otimes \rho_{i}+\bigoplus_{i=1}^{s} \rho_{i}^{\prime} \otimes s o\left(n_{i}\right)
$$

of the group $G \times \mathrm{Sp}\left(2 m_{1}\right) \times \cdots \times \mathrm{Sp}\left(2 m_{k}\right) \times \mathrm{SO}\left(n_{1}\right) \times \cdots \times \mathrm{SO}\left(n_{s}\right)$ (where each copy of $\mathrm{Sp}\left(2 m_{i}\right)$ and of $\mathrm{SO}\left(n_{i}\right)$ acts nontrivially on precisely one irreducible component) is coisotropic if and only if a similar representation of the group $G \times \operatorname{Sp}\left(2 m_{1}^{1}\right) \times \cdots \times$ $\mathrm{Sp}\left(2 m_{k}^{1}\right) \times \mathrm{SO}\left(n_{1}^{1}\right) \times \cdots \times \mathrm{SO}\left(n_{s}^{1}\right)$ with $m_{i}^{1}=\min \left\{\left[\left(p_{i}+1\right) / 2\right], m_{i}\right\}, n_{i}^{1}=\min \left\{p_{i}^{\prime}, n_{i}\right\}$ is coisotropic.

Proof. We need to apply the technique used in the proof of Proposition 10 to each of the representations $\rho_{i} \otimes s p\left(2 m_{i}\right)$ and $\rho_{j}^{\prime} \otimes s o\left(n_{i}\right)$.

2.4.1. Basic reducible coisotropic groups with simple commutator subgroup. Let $G=$ $G^{\prime} \times \mathbb{C}^{* k}$, where the group $G^{\prime}$ is simple and $G: V$ is a coisotropic representation of the group $G$. The representation $G: V$ can be decomposed into the direct sum $V_{1} \oplus V_{2}$, where $G: V_{1}$ is a coisotropic representation that splits in the direct sum of distinct irreducible symplectic representations and $V_{2}$ is a $G$-module of the form $U \oplus U^{*}$, where $G: U$ is a spherical representation.

If the representation $G: V_{1}$ is irreducible, then it is listed in Table 4. Otherwise, it is the direct sum of distinct representations listed in Table 4.

In the second case the representation $G: V_{1}$ is symplectically stable (by Theorem 1), and Table 4 shows that the image of the group $G$ under the representation $G: V_{1}$ is one of the linear groups $\operatorname{Spin}(12)=D_{6}\left(\pi_{5}, \pi_{6}\right), \bigwedge^{3} \mathrm{Sp}(6)$. Now from Table 5 we conclude that $V_{2}=0$. Everywhere later in this subsection we will assume that the representation $G: V_{1}$ is irreducible.

If the representation $G: V$ is coisotropic, then

$$
m_{G^{\prime}}(V)+\operatorname{def}_{G^{\prime}}(V)+2 k \geq \operatorname{dim} V_{1}+2 \operatorname{dim} U,
$$


where $k$ is the dimension of the center of the group $G$.

According to Table 4 each possible group $G^{\prime}$ has one of the following types: $A_{5}, C_{l}$, $l>1, B_{2}, B_{5}, B_{6}, D_{6}, E_{7}$. Now tables 5 and 8 and inequality (2.14) imply that it suffices to consider the following linear groups $G^{\prime} \subset \operatorname{Sp}(V)$ :

(1) $A_{5}\left(\pi_{3}, \pi_{1}, \pi_{5}\right)$

(2) $C_{l}\left(\pi_{1}, \pi_{1}, \pi_{1}\right)$

(3) $C_{l}\left(\pi_{1}, \pi_{1}, \pi_{1}, \pi_{1}, \pi_{1}\right)$,

(4) $C_{3}\left(\pi_{3}, \pi_{1}, \pi_{1}\right)$,

(5) $D_{6}\left(\pi_{5}, \pi_{1}, \pi_{1}\right)$,

(6) $B_{2}\left(\pi_{2}, \pi_{1}, \pi_{1}\right)$,

(7) $A_{5}\left(\pi_{3}, \pi_{1}, \pi_{5}, \pi_{1}, \pi_{5}\right)$.

The group in case (3) is not coisotropic. Indeed, a generic stabilizer for the action $G: V_{2}$ is the subgroup $\operatorname{Sp}(2 l-4) \subset \mathrm{Sp}(2 l)$ with the standard embedding. The restriction of the tautological representation of the group $\operatorname{Sp}(2 l)$ to the subgroup $\operatorname{Sp}(2 l-4)$ is not coisotropic and the desired result follows from Proposition 6 .

Let us prove that the saturated group $G$ with $G^{\prime}=C_{l}\left(\pi_{1}, \pi_{1}, \pi_{1}\right)$ is coisotropic. Using Proposition 14 we see that it suffices to consider the case $l=2$ and to show that

$$
R_{G^{\prime}}(V)=R_{G}(V)=2 m_{G}(V)-\operatorname{dim} V=8 .
$$

The equality $m_{G}(V)=10$ follows easily from the form of a generic stabilizer for the action $G: U \oplus U^{*}$, which can be found using Table 8. Since $m_{G^{\prime}}(V)=9$, we have $R_{G}(V) \leq 9$. On the other hand, $R_{G}(V) \geq 7$ because $2 m_{G}(V)-R_{G}(V) \leq \operatorname{dim} V$. Since the number $R_{G}(V)$ is even, we have $R_{G}(V)=8$. This also implies that the linear group $G^{\prime}$ is not coisotropic.

All other representations are symplectically stable. In case (1) the action $G^{\prime}: V$ is coisotropic by Part 3 of Proposition 1 in Section 1. Using Propositions 6 and 7 in Section 1 applied to a generic stabilizer $H$ for the action $G: V_{1}$, and the classification of spherical representations, we obtain that in cases (4), (5), and (6) the representation $G: V$ is coisotropic. At the same time Proposition 6 implies that in cases (4), (5), (6) the representation $G^{\prime}: V$ is not coisotropic. The same is true for the representation $G: V$ in case (7).

Hence, saturated $A_{1}$-nonglued coisotropic representations with simple commutator subgroup are precisely those listed in Table 10. There is precisely one nonsaturated representation, namely that in the formulation of the theorem.

2.4.2. Coisotropic groups of the form $G=G^{(1)} \otimes G^{(2)}, G^{(1)} \subset \mathrm{Sp}(2 m), G^{(2)} \subset \mathrm{SO}(n)$. Here we classify basic reducible coisotropic representations of the form mentioned in the title. A linear group $G^{(1)} \otimes G^{(2)}$ is reducible if at least one of the linear groups $G^{(1)}, G^{(2)}$ is reducible. In the classification, we omit the cases where the $G$-module $V$ is of the form $U \oplus U^{*}$ for a $G$-module $U$. Also, we omit the cases where the commutator subgroup of $G$ is simple. We have to show that all coisotropic representations of the indicated form are listed in lines 8-11 in Table 10, Let us consider all possible cases.

Case 1. $G=\left(\mathrm{Sp}\left(2 m_{1}\right) \oplus \mathrm{Sp}\left(2 m_{2}\right)\right) \otimes \mathrm{SO}(2 n), n>2$.

By Proposition 14 we can assume that $m_{1}, m_{2} \leq n \leq m_{1}+m_{2}$. Inequality (1.7) can be rewritten in the form

$$
m_{1}^{2}+m_{1}+m_{2}^{2}+m_{2}+n^{2} \geq 2\left(m_{1}+m_{2}\right) n .
$$

For fixed $m_{1}+m_{2}$ the left-hand side of (2.16) attains the maximal value at $m_{1}=n$. Setting $\tilde{m}_{2}=m_{2}+m_{1}-n$, from (2.16) we obtain

$$
\tilde{m}^{2}+\tilde{m}_{2} \geq\left(2 \tilde{m}_{2}-1\right) n .
$$


Clearly, inequality (2.17) can occur only if $\tilde{m}_{2}=0$.

If this is the case, $n=m_{1}+m_{2}$ and (2.16) can be rewritten in the form

$$
m_{1}+m_{2} \geq 2 m_{1} m_{2},
$$

so that $m_{1}=m_{2}=1, n=2$ and we obtain the group in line 8 in Table 10 ,

Case 2. $G=\left(\operatorname{Sp}\left(2 m_{1}\right) \oplus \operatorname{Sp}\left(2 m_{2}\right)\right) \otimes \operatorname{SO}(2 n-1), n>2$.

Similarly to the above, we can assume that $m_{1}, m_{2} \leq n \leq m_{1}+m_{2}$. (In doing so, we skip the group $G=(\mathrm{SL}(2) \oplus \mathrm{SL}(2)) \otimes \mathrm{SO}(2 n-1)$, which, however, was already found in the previous subsection.) Inequality (1.7) can be rewritten in the form

$$
m_{1}^{2}+m_{1}+m_{2}^{2}+m_{2}+(n-1)^{2} \geq\left(m_{1}+m_{2}\right)(2 n-1) .
$$

As before, we see that $m_{1}+m_{2}=n$, and using arguments similar to those presented above we see that the group $G$ is not coisotropic.

Case 3. $G=\mathrm{Sp}(2 m) \otimes\left(\mathrm{SO}\left(n_{1}\right) \oplus \mathrm{SO}\left(n_{2}\right)\right), m>1$.

According to Proposition 14 we can assume that $n_{2} \leq n_{1} \leq 2 m \leq n_{1}+n_{2}+1$. Inequality (1.7) can be rewritten in the form

$$
n_{1}^{2}+n_{2}^{2}+4 m^{2}+4 m \geq 4\left(n_{1}+n_{2}\right) m .
$$

Let $l=n_{1}+n_{2}-2 m \geq 2$. Similarly to Case 1, formula (2.20) implies that

$$
l^{2} \geq 4(l-1) m,
$$

which is impossible for $m>1$.

Let $n_{1}+n_{2}=2 m-1$. In this case $m_{G}=\operatorname{dim} G-1, \operatorname{def}_{G}=\operatorname{rk} G-1$. Indeed, the stabilizer of the generic point for the action $G: V$ is a one-dimensional commutative subalgebra (this follows from Proposition 9 and the characterization of a generic commutative subalgebra in general position for the linear group $\mathrm{Sp}(2 m) \otimes \mathrm{SO}(2 m-1))$. The linear group $G$ is coisotropic if and only if

$$
n_{1}^{2}+n_{2}^{2}-1+4 m(m+1)-4=4(2 m-1) m \Longleftrightarrow n_{1}^{2}+n_{2}^{2}=(2 m-2)^{2}+1,
$$

which holds if and only if $n_{2}=1, n_{1}=2 m-2$.

Let $n_{1}+n_{2}=2 m, 2 m+1$. In this case $m_{G}=\operatorname{dim} G$. Direct verification shows that the group $G$ is coisotropic if and only if $n_{2}=1$.

Therefore, the only family of coisotropic representations of the form under consideration is the family of representations in line 9 in Table 10 .

Case 4. $G=\operatorname{Sp}(2 m)+_{\mathrm{Sp}(2 m)} \mathrm{Sp}(2 m) \otimes G^{(2)}, G^{(2)} \varsubsetneqq \mathrm{SO}(n-1), m>1$.

Denote by $W$ the space of the tautological representation of the group $\operatorname{SO}(n-1)$. Arguments presented in Case 3 above show that the representation $G^{(2)}: W$ cannot be decomposed into the direct sum of two orthogonal representations. Therefore, either $G^{(2)}$ is an irreducible group for which the linear group $\operatorname{Sp}(2 m) \otimes G^{(2)}$ is coisotropic, or $G^{(2)}=G^{(21) \text { ort }}$ for some linear group $G^{(21)}$ such that the representation of the group $\mathrm{Sp}(2 m) \otimes G^{(21)}$ is spherical and irreducible. Denote by $H$ the projection to $\mathrm{Sp}(2 m)$ of a generic stabilizer in the linear group $\operatorname{Sp}(2 m) \otimes G^{(2)}$. The restriction to $H$ of the tautological representation of the group $\operatorname{Sp}(2 m)$ is coisotropic by Proposition 6. In the case where the connected component of this projection is a torus, the converse statement is also true (by Proposition 8). Tables 6 and 8 show that $m=2$ with $G^{(2)}=\operatorname{Spin}(7)$.

Case 5. $G=\left(\operatorname{Sp}\left(2 m_{1}\right) \oplus \operatorname{Sp}\left(2 m_{2}\right)\right) \otimes T(1)$ where $T(1)$ is the image of the embedding of the group $\mathrm{SO}(2)$ into the linear group $\mathrm{SO}(3)$. According to Proposition 14, we can assume that $m_{1}, m_{2} \leq 2$. We have $m_{G}=\operatorname{dim} G-\left(m_{1}+m_{2}-2\right)$, $\operatorname{def}_{G}=\operatorname{rk} G-\left(m_{1}+m_{2}-2\right)$, which imply that $m_{G}+\operatorname{def}_{G}<\operatorname{dim} V$.

Case 6. $G=(\mathrm{SL}(2) \oplus \mathrm{SL}(2)) \otimes G^{(2)}$ where $G^{(2)}$ is a proper subgroup of $S O(n)$. Previous results of this subsection imply that the linear group $G^{(2)}$ is irreducible. Table 6] shows that $G^{(2)}=\operatorname{Spin}(7)$ (for the linear group $G=\operatorname{Sp}(4) \otimes G_{2}\left(\pi_{1}\right)$ a generic stabilizer 
is trivial). Proposition 9 implies that for the linear group $G=(\mathrm{SL}(2) \oplus \mathrm{SL}(2)) \otimes \operatorname{Spin}(7)$, a generic stabilizer is trivial, and Part 2 of Proposition 1 in Section 1 shows that the group $G$ is coisotropic.

Remark 3. Let us note that if a linear group $G=G^{(1)} \otimes G^{(2)}, G^{(1)} \subset \operatorname{Sp}(2 m), G^{(2)} \subset$ $\mathrm{SO}(n)$ (possibly, $A_{1}$-glued) is coisotropic, then its representation does not decompose into the sum of three nontrivial symplectic representations. Indeed, otherwise we have one of the following cases.

(1) The representation of the linear group $G^{(1)}$ can be decomposed into the direct sum of three symplectic representations.

(2) The representation of the linear group $G^{(2)}$ can be decomposed into the direct sum of three orthogonal representations.

(3) The representations of the linear groups $G^{(1)}$ and $G^{(2)}$ both can be decomposed into the direct sum of two symplectic (orthogonal) representations.

The results of the previous classification and the remark after Proposition 13 show that cases (1) and (2) cannot occur. In case (3) we can assume that $G=\left(\operatorname{Sp}\left(2 m_{1}\right) \oplus\right.$ $\left.\mathrm{Sp}\left(2 m_{2}\right)\right) \otimes\left(\mathrm{SO}\left(n_{1}\right) \oplus \mathrm{SO}\left(n_{2}\right)\right)$. Then the previous classification shows that either $m_{1}=$ $m_{2}=1, n_{1}=1$ (which is impossible by Proposition 13) or $n_{1}=n_{2}=2$ (which is impossible by the results of the classification of spherical representations).

Below we will use the following notation. Let $(G, G)=G^{(1)} G^{(2)} \ldots G^{(k)}$ be the decomposition of the commutator subgroup $(G, G)$ of the group $G$ in a locally direct product of simple normal subgroups. Let $\rho$ be a symplectic representation of $G$ whose restriction to $(G, G)$ is decomposed into the direct sum

$$
\rho=\rho_{1}+\cdots+\rho_{l}+\left(\varphi_{1}+\varphi_{1}^{*}\right)+\cdots+\left(\varphi_{m}+\varphi_{m}^{*}\right), \quad l>0, \quad l+m>1,
$$

where the representations $\rho_{i}, \varphi_{j}$ are irreducible and all $\rho_{i}$ are distinct. Let $\rho_{i}=\rho_{i}^{(1)} \otimes$ $\cdots \otimes \rho_{i}^{(k)}, \varphi_{j}=\varphi_{j}^{(1)} \otimes \cdots \otimes \varphi_{j}^{(k)}$, where $\rho_{i}^{(s)}, \varphi_{i}^{(s)}$ are irreducible representations of the simple group $G^{(s)}$.

2.4.3. Coisotropic representations of nonsimple groups that are direct sums of two irreducible components. In this case $l=2, m=0$ (in the notation of (2.22)). Denote by $V_{i}$, $V$ the space of the representation $\rho_{i}, i=1,2$, and $\rho$ respectively.

If $\rho_{1}^{(j)}=\rho_{2}^{(j)}$ for some $j \in \mathbb{N}$, then the representation $\rho$ is of the form studied in the previous subsection. Therefore, we assume that $\rho_{1}^{(j)} \neq \rho_{2}^{(j)}$ for all $j$. We must prove that coisotropic representations of this form are just the representations listed in lines 12-17 in Table 10.

TABle 11. Possible nonzero representations $\rho_{i}^{(j)}, i=1,2$.

\begin{tabular}{|c|c|c|c|c|c|}
\hline$G$ & $B_{l}, l=2,5,6$ & $B_{l}, l=3,4$ & $C_{3}$ & $D_{4}$ & $D_{6}$ \\
\hline $\mathrm{s}$ & $\pi_{l}$ & & $\pi_{1}, \pi_{3}$ & & $\pi_{5}, \pi_{6}$ \\
\hline $\mathrm{o}$ & $\pi_{1}$ & $\pi_{1}, \pi_{l}$ & & $\pi_{1}, \pi_{3}, \pi_{4}$ & $\pi_{1}$ \\
\hline
\end{tabular}

Tables 4 and 6 show that all groups $G^{(j)}$ for which both representations $\rho_{i}^{(j)}$ can be different from the tautological representations, are only those listed in Table 11. The second row contains all possible symplectic representations, and the third row, orthogonal representations. Next, if $\rho_{1}^{(1)}$ is a symplectic representation different from the tautological representation of $\operatorname{Sp}(2 m)$, then $\rho_{1}^{(j)}, j \neq 1$, is the trivial representation, and if $\rho_{1}^{(1)}$ is 
an orthogonal representation, then among the representations $\rho_{1}^{(j)}$ there is at least one tautological representation of the group $\operatorname{Sp}(2 m)$, whereas all other representations are trivial. This follows also from Tables 4 and 6 . Below we consider all possible cases.

Case 1. $\rho_{1}^{(j)}=0$ for $j>1$.

Let $H$ be the connected component of the unit element in a generic stabilizer for the action $G^{(1)}: V_{1}$, and $F$ the projection to $G^{(1)}$ of a generic stabilizer for the action $G: V_{2}$. By $\rho_{0}$ we denote the representation $\rho_{2}^{(2)} \otimes \cdots \otimes \rho_{2}^{(k)}$ of the group $G^{(2)} \times \cdots \times G^{(k)}$.

Tables 4 and 6 show that $\rho_{2}^{(1)}$ should be the tautological representation of one of the groups $\mathrm{Sp}(2 m)$ or $\mathrm{SO}(n)$. Let the linear group $\rho_{1}^{(1)}\left(G^{(1)}\right)$ be one of the groups $C_{3}\left(\pi_{3}\right)$, $B_{5}\left(\pi_{5}\right), B_{6}\left(\pi_{6}\right), D_{6}\left(\pi_{5}\right)$. In this case the restriction of $\rho_{2}^{(1)}$ to $H$ is of the form $\varphi_{0}+\varphi_{0}^{*}$ for the group $G^{(1)}$ of type $C_{3}$ or $D_{6}$, and $\varphi_{0}+\varphi_{0}^{*}+1$ for $G^{(1)}$ of type $B_{5}$ or $B_{6}$. Since the group $H$ is semisimple, $\varphi_{0} \otimes \rho_{0}$ is a spherical representation. Tables 8 and 9 show that the case where $G^{(1)}$ is of type $C_{3}$ does not suit us, and in the remaining cases $\rho_{2}^{(1)}\left(G^{(1)}\right)=\mathrm{SO}(n), n=11,12,13, \rho_{0}(G)=\mathrm{Sp}(2 m)$.

The case where $G^{(1)}$ is the group of type $B_{6}$ cannot occur due to the remark at the end of the previous subsection $\left(\varphi_{0}(H)=\mathrm{SL}(3) \oplus \mathrm{SL}(3)\right.$, see Table 4$)$. In the case where $G^{(1)}$ is of type $B_{5}$, the classification in the previous subsection shows that $\rho_{0}(G)=\mathrm{SL}(2)$, i.e., $G=\mathrm{SL}(2) \otimes \mathrm{SO}(11)+\mathrm{SO}(11) \mathrm{Spin}(11)$. This linear group is coisotropic by Proposition 7 applied to the stabilizer of the representation $G: V_{1}$, and Proposition [13, In the case where $G^{(1)}$ is a group of type $D_{6}$, we have $\varphi_{0}(H)=\mathrm{SL}(6)$ and Table 8 shows that $\rho_{0}(G)=\mathrm{Sp}(2 m), m=1,2$, i.e., $G=\mathrm{Sp}(2 m) \otimes \mathrm{SO}(12)+\mathrm{SO}(12) \operatorname{Spin}_{ \pm}(12)$. The coisotropicity of the linear group $G$ is verified as before.

It remains to consider the case $\rho_{1}^{(1)}\left(G^{(1)}\right)=\operatorname{Sp}(2 m)$. In this case $m=2, \rho_{2}^{(1)}\left(G^{(1)}\right)=$ $\mathrm{SO}(5)$, and $\rho_{0}(G)=\operatorname{Sp}\left(2 m_{1}\right)$. In the case where $m_{1}=m$, the coisotropicity of the group $G$ follows from Proposition 8 .

Let us consider the case where $G=\mathrm{Sp}(4)+{ }_{\mathrm{SO}(5)} \mathrm{SO}(5) \otimes \mathrm{SL}(2)$. In this case $F=$ $\mathrm{SO}(3) \oplus\left(\mathbb{C}^{*}\right)^{\text {ort }}$, so that $m_{G}(V)=\operatorname{dim} G-1$. The action $G: V$ is symplectically stable and applying Part 2 of Proposition 1, we see that the representation $G: V$ is coisotropic.

To prove that linear groups $\mathrm{Sp}(4)+{ }_{\mathrm{Sp}(4)} \mathrm{SO}(5) \otimes \mathrm{Sp}\left(2 m_{1}\right), m_{1}>2$, are coisotropic, it suffices to consider the case $m_{1}=3$ (by Proposition 14). In this case $m_{G}(V)=\operatorname{dim} G-1$, $\operatorname{def}_{G}(V)=\operatorname{rk} G-1$, and $m_{G}(V)+\operatorname{def}_{G}(V)=\operatorname{dim} V$, implying the desired result.

Case 2. $\rho_{2}^{(1)}\left(G^{(1)}\right)=\mathrm{SO}(7), \rho_{1}^{(1)}\left(G^{(1)}\right)=\operatorname{Spin}(7)$. In this case $k \leq 3$.

First let $k=3$, so that $G=\mathrm{Sp}\left(2 m_{1}\right) \otimes \mathrm{SO}(7)+\mathrm{SO}(7) \operatorname{Spin}(7) \otimes \mathrm{Sp}\left(2 m_{2}\right)$. Using the form of a generic stabilizer of the action of the linear group $\operatorname{Sp}\left(2 m_{2}\right) \otimes \operatorname{Spin}(7)$ (Table 6), the results of the previous subsection 2.4.2 and Proposition 6. we conclude that $m_{2}=1$. Similarly, $m_{1}=1$. Coisotropicity of $G$ in this case follows from Proposition 7 .

The case $k=2$ is impossible since in this case we obtain an $A_{1}$-glued group $G$.

Case 3. $\rho_{1}^{(1)}\left(G^{(1)}\right)=\mathrm{SO}(9), \rho_{2}^{(1)}\left(G^{(1)}\right)=\operatorname{Spin}(9)$. Similarly to the above, $k \leq 3$, $G=\mathrm{Sp}\left(2 m_{1}\right) \otimes \mathrm{SO}(9)+{ }_{\mathrm{SO}(9)} \mathrm{Spin}(9) \otimes \mathrm{SL}(2)$. Now from the form of a generic stabilizer of the linear group SL $(2) \otimes \operatorname{Spin}(9)$, results of 2.4.2, and Proposition 6 , we conclude that the linear group $G$ is not coisotropic.

Case 4. $\rho_{1}^{(1)}\left(G^{(1)}\right)=\mathrm{SO}(5), \rho_{2}^{(1)}\left(G^{(1)}\right)=\mathrm{Sp}(4)$.

First we consider the case $G=\mathrm{SO}(n) \otimes \mathrm{Sp}(4)+{ }_{\mathrm{SO}(5)} \mathrm{SO}(5) \otimes \mathrm{Sp}(2 m), n>2$. Using the form of a generic stabilizer for the linear group $\mathrm{Sp}(4) \otimes \mathrm{SO}(n)$, Proposition 6 , and results of 2.4.1, we can conclude that $n=3$. Similarly, $m=1$. Now $\operatorname{dim} G+\operatorname{rk} G=$ $16+4=20 \leq \operatorname{dim} V=22$, contradicting Part 1 of Proposition 1 .

Therefore, in this case there are no coisotropic groups.

Case $5 . G^{(1)}$ is of type $D_{4}$, and $\rho_{1}^{(1)}, \rho_{2}^{(2)}$ have highest weights $\pi_{1}, \pi_{3}$ respectively. Let $k=3$. We have $G=\mathrm{Sp}\left(2 m_{1}\right) \otimes \mathrm{SO}(8)+\mathrm{SO}(8) \operatorname{Spin}_{+}(8) \otimes \mathrm{Sp}\left(2 m_{2}\right)$. 
In the case where $m_{1}>2$, the projection of a generic stabilizer for the action of the linear group $\mathrm{Sp}\left(2 m_{1}\right) \otimes \mathrm{SO}(8)$ on $\mathrm{SO}(8)$ is a torus. Proposition 6 and the classification of spherical representations show that in this case the group $G$ is not coisotropic. Similarly, $m_{2}<3$.

Part 1 of Proposition 1 shows that for $m_{1}=m_{2}=2$ the group $G$ is not coisotropic.

In the case $m_{1}=m_{2}=1$ the group $G$ is coisotropic since this is true even for a smaller group obtained by replacing $\operatorname{SL}(2)$ with $\left(\mathbb{C}^{*}\right)^{\text {sympl }}$ (see Table 9). Therefore, it remains to consider the case $m_{1}=1, m_{2}=2$. To prove that $G$ is coisotropic in this case, one must use Proposition 7 (the linear group which is the image of a generic stabilizer $H$ for the action $G: V_{1}$ under the representation $\rho_{2}$ is $\left.\mathrm{GL}(4)^{\text {ort }} \otimes \operatorname{Sp}(4)\right)$.

The case $k=2$ is impossible due to arguments similar to those used in Case 2.

2.4.4. Remaining coisotropic representations that are direct sums of two minimal representations. Here we classify representations of the form (2.22) with $l=m=1$. Let us consider all possible cases (we consider only $A_{1}$-nonglued representations).

Case 1. The linear group $\rho_{1}(G)$ is simple. Let $H$ be a generic stabilizer for the representation $\rho_{1}^{(1)}$. Then the restriction of $\varphi_{1}$ to $H \otimes G^{(2)} \otimes \cdots \otimes G^{(k)}$ is a spherical representation. Since we do not consider the cases where the group $G^{\prime}=(G, G)$ is simple and where $\varphi_{1}^{(1)}=\rho_{1}^{(1)}$, Tables 4 and 8 show that $\rho_{1}^{(1)}\left(G^{(1)}\right)=\bigwedge^{3} \mathrm{SL}(6)$. Using Table 9 , we obtain the saturated representation $G: V$ listed in line 18 in Table 10. The action $G^{\prime}: V$ is coisotropic as well (by Propositions 7 and 11).

Case 2. $\rho_{1}(G)=\mathrm{SL}(2) \otimes G^{(2)}$. In this case $\varphi^{(1)}=0$ since $\rho$ is assumed to be $A_{1^{-}}$ nonglued. An easy verification using Tables 6 and 8 shows that $G^{(2)}=\mathrm{SO}(n)$ as a linear group. Since $\varphi^{(2)} \neq \rho_{1}^{(2)}$, we have $5 \leq n \leq 10$. Using Propositions 6 and 7 we obtain the saturated representations listed in lines 19-23 in Table 10. The only nonsaturated representation is that of the commutator subgroup of the group in line 19 .

The general case. Here we prove that there are no other coisotropic representations in this class. It suffices to show that $\rho_{1}(G)$ cannot be different from $\operatorname{Sp}(2 m) \otimes G^{(2)}$ where $m>1$, and $G^{(2)}$ cannot be different from $\mathrm{SO}(3)$. One can easily verify that $\varphi^{(1)}=0$. Therefore, it remains to consider the cases $G^{(2)}=\operatorname{SO}(n), n>3$, and $\operatorname{Spin}(7)$. The verification is direct, using Tables 6, 8, and 9,

2.4.5. Coisotropic representations that are sums of three symplectic representations. Let $\rho: G \rightarrow \operatorname{Sp}(V)$ be a saturated representation of this type, $\rho=\rho_{1}+\rho_{2}+\rho_{3}$. According to results of 2.4.1, the commutator subgroup $G^{\prime}$ of the group $G$ is not simple. We can assume that the linear group $G_{12}=\rho_{1}+\rho_{2}(G)$ is one of the groups in lines 8-23 of Table 10. Let us prove that the representation $\rho$ is not coisotropic.

In the case where $G_{12}$ is one of the groups in lines $8,9(2 m \leq n), 10-12,13(m=2)$, $14(m=1), 15,17-21,23$, it suffices to apply Proposition 6 to a generic stabilizer of the representation $\rho_{1}+\rho_{2}$ (or $\rho_{3}$ in cases 7,8$)$ and the remark at the end of 2.4.2.

In case 13 the only possible coisotropic group can be the group $G=(\operatorname{SL}(2) \oplus \operatorname{SL}(2)) \otimes$ $\mathrm{SO}(12)+_{\mathrm{SO}(12)} \mathrm{Spin}_{+}(12)$. To prove that it is not coisotropic as well, it suffices to apply Proposition 6 to the stabilizer of the representation where $G$ acts as $\operatorname{Spin}_{+}(12)$ and the classification results listed in Table 9.

In cases 16, 22 it remains to consider the group $G=\mathrm{SL}(2) \otimes \mathrm{SO}(8)+{ }_{\mathrm{SO}(8)} \mathrm{SL}(2) \otimes$ $\operatorname{Spin}_{+}(8)+{ }_{\mathrm{SO}(8)} \mathrm{SL}(2) \otimes \mathrm{SO}(8)$ only. This group is not coisotropic by Part 1 of Proposition 11.

Finally if $G_{12}$ is a group in lines 9 or 14, the desired result can be obtained by applying Proposition 6 to a generic stabilizer of the representation $\rho_{1}+\rho_{2}$. 


\section{REFERENCES}

[1] E. M. Andreev, E. B. Vinberg, and A. G. Elashvili, Orbits of maximal dimension in semisimple Lie algebras. Funkcional. Anal. i Priložen., 1 (1967), no. 4, 3-7; English transl. in Functional Anal. Appl., 1 (1967). MR0267040 (42:1942)

[2] N. Bourbaki, Groupes et algèbres de Lie. Ch. VII-VIII, Hermann, Paris, 1975. MR0453824 $(56: 12077)$

[3] E. B. Vinberg, Commutative homogeneous spaces and coisotropic symplectic actions. Uspekhi Mat. Nauk, 56 (2001), no. 1(337), 3-62; English transl., Russian Math. Surveys, 56 (2001), no. 1, 1-60. MR0267040 (42:1942)

[4] E. B. Vinberg and A. L. Onishchik, Seminar on Lie groups and Lie algebras. Second edition. URSS, Moscow, 1995; English transl. of the first edition, Lie groups and algebraic groups. Springer-Verlag, Berlin, 1990. MR:1403378 (97d:22001)

[5] E. B. Vinberg and V. L. Popov, Invariant theory. Algebraic geometry, 4, Itogi Nauki i Tekhniki, VINITI, Moscow, 1989, pp. 137-314. (Russian) MR1100485 (92d:14010)

[6] A. G. Elashvili, Canonical form and stationary subalgebras if generic points for simple linear Lie groups. Funkcional. Anal. i Priložen., 6 (1972), no. 1, 51-62; English transl. in Functional Anal. Appl., 6 (1972). MR0304555 (46:3690)

[7] _ Stationary generic subalgebras for irreducible linear Lie groups. Funkcional. Anal. i Priložen. 6 (1972), no. 2, 65-78; English transl. in Functional Anal. Appl., 6 (1972). MR0304554 $(46: 3689)$

[8] C. Benson and G. Ratcliff, Rationality of the generalized binomial coefficients for a multiplicity free action. J. Austral. Math. Society, ser. A. 68 (2000), 387-410. MR.1753368 (2001a:20071)

[9] V. G. Kac, Some remarks on nilpotent orbits. J. Algebra 64 (1980), 190-213. MR0575790 (81i:17005)

[10] A. Leahy, A classification of multiplicity free representations. J. Lie Theory 8 (1998), 367-391. MR.1650378 (2000g:22024)

2Nd Bagration Per. 19-706, Minsk 220037, Belarus

E-mail address: ivanlosev@yandex.ru

Translated by O. KHLEBORODOVA 